\title{
REPRESENTATIONS OF AN INTEGER BY SOME QUATERNARY AND OCTONARY QUADRATIC FORMS
}

\author{
B. RAMAKRISHNAN, BRUNDABAN SAHU AND ANUP KUMAR SINGH \\ Dedicated to Professor V. Kumar Murty on the occasion of his 60th birthday
}

\begin{abstract}
In this paper we consider certain quaternary quadratic forms and octonary quadratic forms and by using the theory of modular forms, we find formulae for the number of representations of a positive integer by these quadratic forms.
\end{abstract}

\section{INTRODUCTION}

In this paper we consider two types of quadratic forms, viz., quaternary and octonary forms. In the first part, we deal with quaternary quadratic forms of the following type given by $\mathcal{Q}_{a, \ell}=\mathcal{Q}_{a} \oplus \ell \mathcal{Q}_{a}: x_{1}^{2}+x_{1} x_{2}+a x_{2}^{2}+\ell\left(x_{3}^{2}+x_{3} x_{4}+a x_{4}^{2}\right)$, where $\mathcal{Q}_{a}$ is the quadratic form $x_{1}^{2}+x_{1} x_{2}+a x_{2}^{2}$. Let $R_{a, \ell}(n)$ denote the number of ways of representing a positive integer $n$ by the quadratic form $\mathcal{Q}_{a, \ell}$. i.e.,

$$
R_{a, \ell}(n):=\operatorname{card}\left\{\left(x_{1}, x_{2}, x_{3}, x_{4}\right) \in \mathbb{Z}^{4}: n=x_{1}^{2}+x_{1} x_{2}+a x_{2}^{2}+\ell\left(x_{3}^{2}+x_{3} x_{4}+a x_{4}^{2}\right)\right\} .
$$

One of the main results of this paper is to find formulas for $R_{a, \ell}(n),(a, \ell) \in \mathbf{A}$, where $\mathbf{A}=$ $\{(1,2),(1,3),(1,4),(1,5),(2,2),(2,3),(2,4),(3,2),(3,3),(4,2),(5,1),(5,2)\}$. Let us mention a brief account of similar results obtained so far. S. Ramanujan was the first to observe the following identity:

$$
\left(\sum_{m=-\infty}^{\infty} \sum_{n=-\infty}^{\infty} q^{m^{2}+m n+n^{2}}\right)^{2}=-\frac{1}{2} E_{2}(z)+\frac{3}{2} E_{2}(3 z) .
$$

(See [6, pp. 402-403], [7, p.460, Entry 3.1] for details.) Since

$$
\left(\sum_{m=-\infty}^{\infty} \sum_{n=-\infty}^{\infty} q^{m^{2}+m n+n^{2}}\right)^{2}=1+\sum_{n=1}^{\infty} R_{1,1}(n) q^{n}
$$

by comparing the $n$-th Fourier coefficients in (1), one gets

$$
R_{1,1}(n)=12 \sigma(n)-36 \sigma(n / 3) .
$$

In the above, $E_{2}(z)$ denotes the Eisenstein series of weight 2 on $S L_{2}(\mathbb{Z})$ which is given by

$$
E_{2}(z)=1-24 \sum_{n \geq 1} \sigma(n) q^{n} .
$$

Note that $E_{2}(z)$ is a quasimodular form. Here $q=e^{2 \pi i z}, z \in \mathbb{H}$, where $\mathbb{H}=\{z \in \mathbb{C}: \operatorname{Im}(z)>0\}$. We remark that Ramanujan gave the identity (1) without proof. For a proof of (2) one can refer to Huard et al. [[11], Theorem 13] or Lomadze[10]. Formulas for $R_{a, 1}(n)$ for $a=2,3,4,6,7$ are known due to the works of several authors using different methods. In this paper, we consider the case

Date: May 14, 2019.

2010 Mathematics Subject Classification. Primary 11E25, 11F11; Secondary 11E20.

Key words and phrases. quaternary quadratic forms; octonary quadratic forms; modular forms of one variable, classical theta function, convolution sums of the divisor functions. 
$a=5$. Further, we also consider the case $\ell>1$ for a few values of $\ell$. More precisely, for the pairs $(a, \ell)$ belonging to the set $\mathbf{A}$. In the following table, we list the present work and also the earlier works done in this direction.

\begin{tabular}{|c|c|c|c|}
\hline $\begin{array}{c}\text { Present work } \\
(a, \ell)\end{array}$ & $\begin{array}{c}\text { Earlier works } \\
(a, 1)\end{array}$ & $\begin{array}{c}\text { Author(s) } \\
(\text { earlier works })\end{array}$ & References \\
\hline \hline$(1,2),(1,3),(1,4),(1,5)$ & $(1,1)$ & Huard et al., Lomadze & {$[1],[13]$} \\
\hline$(2,2),(2,3),(2,4)$ & $(2,1)$ & Ramanujan, Chan-Ong, Williams & {$[\underline{6},[$,, 22$]$} \\
\hline$(3,2),(3,3)$ & $(3,1)$ & Chan-Cooper & {$[8]$} \\
\hline$(4,2)$ & $(4,1)$ & Cooper-Ye & {$[10]$} \\
\hline$(5,1),(5,2)$ & - & - & - \\
\hline- & $(6,1)$ & Chan-Cooper & {$[8]$} \\
\hline- & $(7,1)$ & Dongxi Ye & {$[23]$} \\
\hline
\end{tabular}

Table 1.

Some of the formulas for $R_{a, \ell}(n)$ involve only the divisor function $\sigma(n)$, namely the cases $(a, \ell)=$ $(1,2),(1,4),(3,2)$. In these cases it is possible to get formulas for the number of representations of the quadratic forms in eight variables defined by $\mathcal{Q}_{a, \ell ; j}:=\mathcal{Q}_{a, \ell} \oplus j \mathcal{Q}_{a, \ell}$ using the convolution sums method. We note that this method (doubling the quadratic form with coefficients) can be considered in general, here for simplicity we have considered only the following 7 cases: $(a, \ell, j)=$ $(1,2,1),(1,2,2),(1,2,3),(1,2,4),(1,4,1),(1,4,2),(3,2,1)$. To be precise, in these cases mentioned above, the formulas do not involve too many coefficients coming from the cusp forms.

In the second part of this article, we consider the following octonary quadratic forms (with coefficients $1,2,4,8)$ :

$$
\sum_{r=1}^{i} x_{r}^{2}+2 \sum_{r=i+1}^{i+j} x_{r}^{2}+4 \sum_{r=i+j+1}^{i+j+k} x_{r}^{2}+8 \sum_{r=i+j+k+1}^{i+j+k+l} x_{r}^{2}
$$

for all partitions $i+j+k+l=8, i, j, k, l \geq 0$. There are a total of 165 such quadratic forms, and out of which 81 quadratic forms (corresponding to $i=0$ or $l=0$ ) have already been considered by several authors [3, 4, 17. In the second part, we consider the remaining 84 quadratic forms and give formulas for the corresponding representation numbers. All these 84 quadratic forms are listed as quadruples $(i, j, k, l)$ (corresponding to $i \neq 0$ and $l \neq 0$ ) in Table 2 below.

\begin{tabular}{|r|c|}
\hline$(i, j, k, l)$ & Type \\
\hline $\begin{array}{r}(1,0,1,6),(1,0,3,4),(1,0,5,2),(1,1,1,5),(1,1,3,3),(1,1,5,1),(1,2,1,4),(1,2,3,2),(1,3,1,3),(1,3,3,1), \\
(1,4,1,2),(1,5,1,1),(2,0,0,6),(2,0,2,4),(2,0,4,2),(2,1,0,5),(2,1,2,3),(2,1,4,1),(2,2,0,4),(2,2,2,2),\end{array}$ & I \\
$(2,3,0,3),(2,3,2,1),(2,4,0,2),(2,5,0,1),(3,0,1,4),(3,0,3,2),(3,1,1,3),(3,1,3,3),(3,2,1,2),(3,3,1,1)$, & \\
$(4,0,0,4),(4,0,2,2),(4,1,0,3),(4,1,2,1),(4,2,0,2),(4,3,0,1),(5,0,1,2),(5,1,1,1),(6,0,0,2),(6,1,0,1)$ & \\
& \\
& \\
& \\
$(1,0,0,7),(1,0,2,5),(1,0,4,3),(1,0,6,1),(1,1,0,6),(1,1,2,4),(1,1,4,2),(1,2,0,5),(1,2,2,3),(1,2,4,1)$, & \\
$(1,3,0,4),(1,3,2,2),(1,4,0,3),(1,4,2,1),(1,5,0,2),(1,6,0,1),(2,0,1,5),(2,0,3,3),(2,0,5,1),(2,1,1,4)$, & II \\
$(2,1,3,2),(2,2,1,3),(2,2,3,1),(2,3,1,2),(2,4,1,1),(3,0,0,5),(3,0,2,3),(3,0,4,1),(3,1,0,4),(3,1,2,2)$, & \\
$(3,2,0,3),(3,2,2,1),(3,3,0,2),(3,4,0,1),(4,0,1,3),(4,0,3,1),(4,1,1,2),(4,2,1,1),(5,0,0,3),(5,0,2,1)$, & \\
$(5,1,0,2),(5,2,0,1),(6,0,1,1),(7,0,0,1)$ & \\
\hline
\end{tabular}

Table 2.

There are several methods used in the literature to obtain results of this type. In this paper, we use the theory of modular forms to prove our formulas. We first obtain the level and character of the modular forms corresponding to these quadratic forms. Then by using explicit bases for the spaces of modular forms, we deduce our formulas. 


\section{Preliminaries and statement of Results}

As we use the theory of modular forms, we shall first present some preliminary facts on modular forms. For $k \in \frac{1}{2} \mathbb{Z}$, let $M_{k}\left(\Gamma_{0}(N), \chi\right)$ denote the space of modular forms of weight $k$ for the congruence subgroup $\Gamma_{0}(N)$ with character $\chi$ and $S_{k}\left(\Gamma_{0}(N), \chi\right)$ be the subspace of cusp forms of weight $k$ for $\Gamma_{0}(N)$ with character $\chi$. We assume $4 \mid N$ when $k$ is not an integer and in that case, the character $\chi$ which is a Dirichlet character modulo $N$, is an even character. When $\chi$ is the trivial (principal) character modulo $N$, we shall denote the spaces by $M_{k}\left(\Gamma_{0}(N)\right)$ and $S_{k}\left(\Gamma_{0}(N)\right)$ respectively. Further, when $k \geq 4$ is an integer and $N=1$, we shall denote the vector spaces by $M_{k}$ and $S_{k}$ respectively.

For an integer $k \geq 4$, let $E_{k}$ denote the normalized Eisenstein series of weight $k$ in $M_{k}$ given by

$$
E_{k}(z)=1-\frac{2 k}{B_{k}} \sum_{n \geq 1} \sigma_{k-1}(n) q^{n}
$$

where $q=e^{2 i \pi z}, \sigma_{r}(n)$ is the sum of the $r$ th powers of the positive divisors of $n$, and $B_{k}$ is the $k$-th Bernoulli number defined by $\frac{x}{e^{x}-1}=\sum_{m=0}^{\infty} \frac{B_{m}}{m !} x^{m}$.

The classical theta function which is fundamental to the theory of modular forms of half-integral weight is defined by

$$
\Theta(z)=\sum_{n \in \mathbb{Z}} q^{n^{2}}
$$

and is a modular form in the space $M_{1 / 2}\left(\Gamma_{0}(4)\right)$. Another function which is mainly used in our work is the Dedekind eta function $\eta(z)$, which is defined by

$$
\eta(z)=q^{1 / 24} \prod_{n \geq 1}\left(1-q^{n}\right) .
$$

An eta-quotient is a finite product of integer powers of $\eta(z)$ and we denote it as follows.

$$
\prod_{i=1}^{s} \eta^{r_{i}}\left(d_{i} z\right):=d_{1}^{r_{1}} d_{2}^{r_{2}} \cdots d_{s}^{r_{s}}
$$

where $d_{i}$ 's are positive integers and $r_{i}$ 's are non-zero integers.

Suppose that $\chi$ and $\psi$ are primitive Dirichlet characters with conductors $M$ and $N$, respectively. For a positive integer $k$, let

$$
E_{k, \chi, \psi}(z):=c_{0}+\sum_{n \geq 1}\left(\sum_{d \mid n} \psi(d) \cdot \chi(n / d) d^{k-1}\right) q^{n},
$$

where

$$
c_{0}= \begin{cases}0 & \text { if } M>1, \\ -\frac{B_{k, \psi}}{2 k} & \text { if } M=1,\end{cases}
$$

and $B_{k, \psi}$ denotes generalized Bernoulli number with respect to the character $\psi$. Then, the Eisenstein series $E_{k, \chi, \psi}(z)$ belongs to the space $M_{k}\left(\Gamma_{0}(M N), \chi / \psi\right)$, provided $\chi(-1) \psi(-1)=(-1)^{k}$ and $M N \neq$ 1. When $\chi=\psi=1$ (i.e., when $M=N=1$ ) and $k \geq 4$, we have $E_{k, \chi, \psi}(z)=E_{k}(z)$, the normalized Eisenstein series of integer weight $k$ as defined before. We refer to [14, 21] for details. We give a notation to the inner sum in (8):

$$
\sigma_{k-1 ; \chi, \psi}(n):=\sum_{d \mid n} \psi(d) \cdot \chi(n / d) d^{k-1}
$$


Let $\mathbb{N}$ and $\mathbb{N}_{0}$ denote the set of positive integers and non-negative integers respectively. For $a_{1}, \ldots, a_{8} \in \mathbb{N}$ and $n \in \mathbb{N}_{0}$, we define

$$
N\left(a_{1}, \ldots, a_{8} ; n\right):=\operatorname{card}\left\{\left(x_{1}, \ldots, x_{8}\right) \in \mathbb{Z}^{8} \mid n=a_{1} x_{1}^{2}+\cdots+a_{8} x_{8}^{2}\right\} .
$$

Note that $N\left(a_{1}, \ldots, a_{8} ; 0\right)=1$. Without loss of generality we may assume that

$$
a_{1} \leq a_{2} \leq \cdots \leq a_{8} \text { and } \operatorname{gcd}\left(a_{1}, \ldots, a_{8}\right)=1 .
$$

In our work, we assume that $a_{1}, \ldots, a_{8} \in\{1,2,4,8\}$. For the octonary quadratic forms given by (4), the number of representations is denoted (in the above notation) by $N\left(1^{i}, 2^{j}, 4^{k}, 8^{l} ; n\right), i+j+k+l=8$. In our earlier paper [17, we had listed some of the basic results in the theory of modular forms of integral and half-integral weight, which will be used in our proof. For more details we refer to [12, 14, 20].

We now list the main results of this paper.

Theorem 2.1. For $n \in \mathbb{N}$, we have

$$
\begin{aligned}
R_{1,2}(n)= & 6 \sigma(n)-12 \sigma(n / 2)+18 \sigma(n / 3)-36 \sigma(n / 6) \\
R_{1,3}(n)= & 3 \sigma(n)-27 \sigma(n / 9)+3 k(n) \\
R_{1,4}(n)= & 6 \sigma(n)-18 \sigma(n / 2)-18 \sigma(n / 3)+24 \sigma(n / 4)+54 \sigma(n / 6)-72 \sigma(n / 12) \\
R_{1,5}(n)= & \frac{3}{2} \sigma(n)+\frac{9}{2} \sigma(n / 3)-\frac{15}{2} \sigma(n / 5)-\frac{45}{2} \sigma(n / 15)+\frac{9}{2} \tau_{2,15}(n) \\
R_{2,2}(n)= & \frac{4}{3} \sigma(n)+\frac{8}{3} \sigma(n / 2)-\frac{28}{3} \sigma(n / 7)-\frac{56}{3} \sigma(n / 14)+\frac{2}{3} \tau_{2,14}(n) \\
R_{2,3}(n)= & \frac{63}{40} \sigma(n)-\frac{9}{2} \sigma(n / 3)+\frac{21}{2} \sigma(n / 7)-\frac{1323}{40} \sigma(n / 21)+\frac{1}{2} \tau_{2,21}(n) \\
R_{2,4}(n)= & \frac{2}{3} \sigma(n)+\frac{2}{3} \sigma(n / 2)+\frac{8}{3} \sigma(n / 4)-\frac{14}{3} \sigma(n / 7)-\frac{14}{3} \sigma(n / 14)-\frac{56}{3} \sigma(n / 28) \\
& +\frac{4}{3} \tau_{2,14}(n)+\frac{8}{3} \tau_{2,14}(n / 2) \\
R_{3,2}(n)= & 2 \sigma(n)-4 \sigma(n / 2)+22 \sigma(n / 11)-44 \sigma(n / 22) \\
R_{3,3}(n)= & \frac{3}{5} \sigma(n)+\frac{9}{5} \sigma(n / 3)-\frac{33}{5} \sigma(n / 11)-\frac{99}{5} \sigma(n / 33)+\frac{16}{15} \tau_{2,11}(n) \\
& +\frac{16}{5} \tau_{2,11}(n / 3)+\frac{1}{3} \tau_{2,33}(n) \\
& \frac{1}{2} \sigma(n)+\sigma(n / 2)+\frac{3}{2} \sigma(n / 3)-\frac{5}{2} \sigma(n / 5)+3 \sigma(n / 6)-5 \sigma(n / 10)-\frac{15}{2} \sigma(n / 15) \\
R_{4,2}(n) & -15 \sigma(n / 30)+\frac{1}{2} \tau_{2,15}(n)+\tau_{2,15}(n / 2)+\tau_{2,30}(n) \\
R_{5,1}(n)= & \frac{4}{3} \sigma(n)-\frac{76}{3} \sigma(n / 19)+\frac{8}{3} \tau_{2,19}(n) \\
R_{5,2}(n)= & \frac{6}{5} \sigma(n)-\frac{12}{5} \sigma(n / 2)+\frac{114}{5} \sigma(n / 19)-\frac{228}{5} \sigma(n / 38)+\frac{4}{5} \tau_{2,38 ; 2}(n) . \\
= &
\end{aligned}
$$

Note: In the above theorem, $k(n)$ denotes the $n$-th Fourier coefficient of the eta-quotient $\frac{\eta^{3}(z) \eta^{3}(9 z)}{\eta^{2}(3 z)}$ and $\tau_{k, N}(n)$ denotes the $n$-th Fourier coefficient of the normalized newform in the space $S_{k}\left(\Gamma_{0}(N), \chi\right)$. Also, if there are more than one newform, then $\tau_{k, N ; j}(n)$ is the $n$-th Fourier coefficient of the $j$-th newform.

As mentioned in the introduction, since the formulas for $R_{1,2}(n), R_{1,4}(n)$ and $R_{3,2}(n)$ involve only the divisor function $\sigma(n)$, we use the convolution sums of the divisor functions to get formulas for 
a few more quadratic forms in eight variables, namely, the quadratic forms defined by $\mathcal{Q}_{a, \ell} \oplus j \mathcal{Q}_{a, \ell}$, which is denoted by $\mathcal{Q}_{a, \ell ; j}$. Let $R_{a, \ell ; j}(n)$ be the number of representations of $n$ by this quadratic form. In Theorem 2.2. we give formulas for $R_{a, \ell ; j}(n)$ when $(a, \ell, j)=(1,2,1),(1,2,2),(1,2,3),(1,2,4)$, $(1,4,1),(1,4,2),(3,2,1)$. In order to get these formulas we need the convolution sums $W_{a, b}(n)$, $(a, b)=$ and $W_{N}(n)$, for $1 \leq N \leq 24$. Here the convolution sums are defined as follows:

$$
W_{a, b}(n)=\sum_{a i+b j=n} \sigma(i) \sigma(j) .
$$

We write $W_{1, N}(n)$ and $W_{N, 1}(n)$ as $W_{N}(n)$. Also note that $W_{a, b}(n)=W_{b, a}(n)$. In all the above convolution sums, the indices used are natural numbers. The following theorem gives the representation numbers $R_{a, \ell ; j}(n)$ for the above mentioned triplets $(a, \ell, j)$.

\section{Theorem 2.2.}

$$
\begin{aligned}
R_{1,2 ; 1}(n)= & \frac{24}{5} \sigma_{3}(n)+\frac{96}{5} \sigma_{3}(n / 2)+\frac{216}{5} \sigma_{3}(n / 3)+\frac{864}{5} \sigma_{3}(n / 6)+\frac{36}{5} \tau_{4,6}(n), \\
R_{1,2 ; 2}(n)= & \frac{12}{5} \sigma_{3}(n)-\frac{84}{5} \sigma_{3}(n / 2)+\frac{108}{5} \sigma_{3}(n / 3)+\frac{192}{5} \sigma_{3}(n / 4)-\frac{756}{5} \sigma_{3}(n / 6) \\
& +\frac{1728}{5} \sigma_{3}(n / 12)+\frac{18}{5} \tau_{4,6}(n)+\frac{72}{5} \tau_{4,6}(n / 2), \\
R_{1,2 ; 3}(n)= & \frac{2}{5} \sigma_{3}(n)+\frac{8}{5} \sigma_{3}(n / 2)+\frac{76}{5} \sigma_{3}(n / 3)+\frac{304}{5} \sigma_{3}(n / 6)+\frac{162}{5} \sigma_{3}(n / 9) \\
& +\frac{648}{5} \sigma_{3}(n / 18)+6(n+1) \sigma(n)+\frac{3}{5} \tau_{4,6}(n)+\frac{27}{5} \tau_{4,6}(n / 3)-2 \tau_{4,9}(n) \\
& -8 \tau_{4,9}(n / 2)+\frac{1}{5} c_{2,9}(n)+\frac{31}{5} c_{1,18}(n) \\
& \frac{33}{40} \sigma_{3}(n)-\frac{93}{40} \sigma_{3}(n / 2)+\frac{297}{40} \sigma_{3}(n / 3)-\frac{93}{10} \sigma_{3}(n / 4)-\frac{837}{40} \sigma_{3}(n / 6) \\
& +\frac{264}{5} \sigma_{3}(n / 8)-\frac{837}{10} \sigma_{3}(n / 12)+\frac{2376}{5} \sigma_{3}(n / 24)+\left(18-\frac{27}{2} n\right) \sigma(n / 3) \\
& +27(1-n) \sigma(n / 8)-\frac{27}{10} \tau_{4,6}(n)-18 \tau_{4,6}(n / 2)-\frac{216}{5} \tau_{4,6}(n / 4) \\
& +\frac{9}{8} \tau_{4,8}(n)+\frac{81}{8} \tau_{4,8}(n / 3), \\
& \frac{6}{5} \sigma_{3}(n)+\frac{18}{5} \sigma_{3}(n / 2)+\frac{54}{5} \sigma_{3}(n / 3)+\frac{96}{5} \sigma_{3}(n / 4)+\frac{162}{5} \sigma_{3}(n / 6) \\
& +\frac{864}{5} \sigma_{3}(n / 12)-36 \sigma(n / 6)+\frac{54}{5} \tau_{4,6}(n)+\frac{216}{5} \tau_{4,6}(n / 2), \\
& \frac{3}{5} \sigma_{3}(n)+\frac{39}{5} \sigma_{3}(n / 2)+\frac{27}{5} \sigma_{3}(n / 3)+\frac{102}{5} \sigma_{3}(n / 4)+\frac{351}{5} \sigma_{3}(n / 6) \\
& +\frac{1056}{5} \sigma_{3}(n / 8)-\frac{1242}{5} \sigma_{3}(n / 12)+\frac{864}{5} \sigma_{3}(n / 24)+54(4-n) \sigma(n / 2) \\
R_{1,4 ; 1}(n)=\frac{9}{10} \tau_{4,6}(n) & (n / 2)-\frac{1872}{5} \tau_{4,6}(n / 4)-\frac{9}{4} \tau_{4,8}(n)-\frac{81}{4} \tau_{4,8}(n / 3)+\frac{6}{40} c_{3,8}(n) \\
R_{1,4 ; 2}(n)=(n / 8)-18(13+6 n) \sigma(n / 12)-540 n \sigma(n / 24)-12(n / 2)+\frac{549}{40} c_{1,24}(n) . & \\
= & \\
= &
\end{aligned}
$$




$$
\begin{aligned}
R_{3,2 ; 1}(n)= & \frac{24}{61} \sigma_{3}(n)+\frac{96}{61} \sigma_{3}(n / 2)+\frac{2904}{61} \sigma_{3}(n / 11)+\frac{11616}{61} \sigma_{3}(n / 22)+\frac{220}{61} a_{1}(n) \\
& -\frac{480}{61} a_{1}(n / 2)+\frac{1976}{61} a_{2}(n)-\frac{3296}{61} a_{2}(n / 2)+\frac{6276}{61} a_{3}(n)-\frac{7680}{61} a_{3}(n / 2) \\
& +\frac{9280}{61} a_{4}(n)-\frac{7680}{61} a_{4}(n / 2)+\frac{5440}{61} a_{5}(n) .
\end{aligned}
$$

Remark 2.1. As mentioned before, $\tau_{k, N}(n)$ denotes the $n$-th Fourier coefficient of the newform of weight $k$, level $N$. The coefficients $c_{2,9}(n), c_{1,18}(n)$ were defined in [1, Definition 2.1] and the coefficients $c_{3,8}(n), c_{1,24}(n)$ were defined in [2, Definition 2.1]. The remaining coefficients $a_{j}(n)$ that appear in the above formulas are defined by the equations (45) to (51).

The next theorem gives the formulae for the octonary quadratic forms with coefficients $1,2,4$, and 8 given in Table 2. We present them as two statements, each statement corresponds to the two modular forms spaces $\left(M_{4}\left(\Gamma_{0}(32)\right)\right.$ for Type I and $M_{4}\left(\Gamma_{0}(32), \chi_{8}\right)$ for Type II $)$ that appear in Table 2 respectively.

Theorem 2.3. Let $n \in \mathbb{N}$ and $i, j, k, l$ be non-negative integers such that $i+j+k+l=8$.

(i) For each entry $(i, j, k, l)$ in Table 2 corresponding to the space $M_{4}\left(\Gamma_{0}(32)\right)$, i.e. $j+l \equiv 0(2)$, we have

$$
N\left(1^{i}, 2^{j}, 4^{k}, 8^{l} ; n\right)=\sum_{\alpha=1}^{16} c_{\alpha} C_{\alpha}(n)
$$

where $C_{\alpha}(n)$ are the Fourier coefficients of the basis elements $F_{\alpha}$ defined in $\S 4.4$ and the values of the constants $c_{\alpha}$ are given in Table 3 .

(ii) For each entry $(i, j, k, l)$ in Table 2 corresponding to the space $M_{4}\left(\Gamma_{0}(32), \chi_{8}\right)$, i.e., $j+l \equiv 1(2)$, we have

$$
N\left(1^{i}, 2^{j}, 4^{k}, 8^{l} ; n\right)=\sum_{\alpha=1}^{16} d_{\alpha} D_{\alpha}(n),
$$

where $D_{\alpha}(n)$ are the Fourier coefficients of the basis elements $G_{\alpha}$ defined in $\S 4.5$ and the values of the constants $d_{\alpha}$ are given in Table 4.

\section{SAMPLE FORMULAS}

In this section we shall give explicit formulas for a few cases of (30) and (31) in Theorem 2.3. We first give the formulas for the cases $(1,0,1,6)$ and $(1,1,1,5)$ in Table 2 (Type I), which correspond to the space $M_{4}\left(\Gamma_{0}(32)\right)$.

For $n \in \mathbb{N}$, we have

$$
\begin{aligned}
N\left(1^{1}, 4^{1}, 8^{6} ; n\right)= & \frac{1}{64} \sigma_{3}(n)-\frac{9}{64} \sigma_{3}(n / 2)+\frac{17}{8} \sigma_{3}(n / 4)-2 \sigma_{3}(n / 8)-16 \sigma_{3}(n / 16) \\
& +256 \sigma_{3}(n / 32)+\frac{1}{64} \sigma_{3 ; \chi-4, \chi-4}(n)+\frac{31}{64} a_{4,8}(n)+2 a_{4,8}(n / 4)+\frac{31}{64} a_{4,16}(n) \\
& +\frac{13}{8} a_{4,32,1}(n)+\frac{3}{4} a_{4,32,2}(n)-\frac{5}{8} a_{4,32,3}(n), \\
N\left(1^{1}, 2^{1}, 4^{1}, 8^{5} ; n\right)= & \frac{1}{32} \sigma_{3}(n)-\frac{1}{32} \sigma_{3}(n / 2)-16 \sigma_{3}(n / 16)-256 \sigma_{3}(n / 32)+\frac{11}{32} a_{4,8}(n) \\
& +\frac{3}{4} a_{4,8}(n / 2)+2 a_{4,8}(n / 4)+\frac{5}{8} a_{4,16}(n)+\frac{11}{8} a_{4,32,1}(n)+\frac{1}{4} a_{4,32,2}(n) \\
& -\frac{3}{8} a_{4,32,3}(n) .
\end{aligned}
$$


Next we give the formulas for the cases $(1,0,0,7)$ and $(1,1,2,4)$ in Table 2 (Type II), which correspond to the space $M_{4}\left(\Gamma_{0}(32), \chi_{8}\right)$.

For $n \in \mathbb{N}$, we have

$$
\begin{aligned}
N\left(1^{1}, 8^{7} ; n\right)= & \frac{1}{88} \sigma_{3, \chi_{0}, \chi_{2}}(n)-\frac{1}{88} \sigma_{3, \chi_{0}, \chi_{2}}(n / 2)-\frac{2}{11} \sigma_{3, \chi_{0}, \chi_{2}}(n / 4)+\frac{1}{88} \sigma_{3, \chi_{2}, \chi_{0}}(n) \\
& -\frac{1}{11} \sigma_{3, \chi_{2}, \chi_{0}}(n / 2)-\frac{16}{11} \sigma_{3, \chi_{2}, \chi_{0}}(n / 4)+\frac{1}{88} \sigma_{3 ; \chi_{-4}, \chi_{-8}}(n)+\frac{1}{88} \sigma_{3 ; \chi_{-8}, \chi_{-4}}(n) \\
& +\frac{43}{176} a_{4,8, \chi_{8} ; 1}(n)+\frac{43}{22} a_{4,8, \chi_{8} ; 1}(n / 2)+\frac{8}{11} a_{4,8, \chi_{8} ; 1}(n / 4)-\frac{129}{176} a_{4,8, \chi_{8} ; 2}(n) \\
& -\frac{43}{44} a_{4,8, \chi_{8} ; 2}(n / 2)-\frac{4}{11} a_{4,8, \chi_{8} ; 2}(n / 4)+\frac{43}{44} a_{4,32, \chi_{8} ; 1}(n)+\frac{43}{44} a_{4,32, \chi_{8} ; 2}(n), \\
N\left(1^{1}, 2^{1}, 4^{2}, 8^{4} ; n\right)= & \frac{2}{11} \sigma_{3, \chi_{0}, \chi_{2}}(n / 4)+\frac{1}{22} \sigma_{3, \chi_{2}, \chi_{0}}(n)+\frac{3}{22} a_{4,8, \chi_{8} ; 1}(n)+2 a_{4,8, \chi_{8} ; 1}(n / 2) \\
& -\frac{48}{11} a_{4,8, \chi_{8} ; 1}(n / 4)-\frac{9}{11} a_{4,8, \chi_{8} ; 2}(n)+a_{4,8, \chi_{8} ; 2}(n / 2)+\frac{16}{11} a_{4,8, \chi_{8} ; 2}(n / 4) \\
& +a_{4,32, \chi_{8} ; 1}(n)+2 a_{4,32, \chi_{8} ; 2}(n) .
\end{aligned}
$$

\section{Proofs of Theorems}

4.1. Proof of Theorem 2.1. Let $\Theta_{a, \ell}(z)$ denote the theta series associated to the quadratic form $\mathcal{Q}_{a, \ell}$. Then

$$
\Theta_{a, \ell}(z)=\Theta_{a}(z) \Theta_{a}(\ell z)
$$

where $\Theta_{a}(z)$ is the theta function associated to the quadratic form $\mathcal{Q}_{a}$. i.e.,

$$
\Theta_{a}(z)=\sum_{m, n=-\infty}^{\infty} q^{m^{2}+m n+a n^{2}} .
$$

Recall $q=e^{2 \pi i z}$. Since $R_{a, \ell}(n)$ is the number of representations of a positive integer $n$ by the quadratic form $\mathcal{Q}_{a, \ell}$, we see that

$$
\Theta_{a, \ell}(z)=1+\sum_{n=1}^{\infty} R_{a, \ell}(n) q^{n} .
$$

So, it is sufficient to write the theta series $\Theta_{a, \ell}(z)$ in terms of a basis of the space of modular forms in order to get our formulas.

Lemma 4.1. The theta series $\Theta_{a, \ell}(z)$ is a modular form of weight 2 on $\Gamma_{0}(\operatorname{lcm}[\ell,(4 a-1)])$ with trivial character.

Proof. By [19, Theorem 4], it follows that $\Theta_{a}(z)$ is a modular form of weight 1 on $\Gamma_{0}(4 a-1)$ with character $\left(\frac{\cdot}{4 a-1}\right)$. Also, it is a well-known fact that if $f$ is a modular form of integer weight $k$ on $\Gamma_{0}(N)$ with character $\psi$, then for a positive integer $d$, the function $f(d z)$ is a modular form of same weight $k$ on $\Gamma_{0}(d N)$ with character $\psi$. Further, if $f_{i}$ are modular forms of weight $k_{i}$, on $\Gamma_{0}\left(N_{i}\right)$ with chracter $\psi_{i}, i=1,2$, then the product $f_{1} f_{2}$ is a modular form of weight $k_{1}+k_{2}$ on $\Gamma_{0}\left(\operatorname{lcm}\left[N_{1}, N_{2}\right]\right)$ with character $\psi_{1} \psi_{2}$. For these facts, we refer to [12, Chapter 3]. We also refer to the proof of Fact II in our earlier work [17, which contains details of the above arguments. Therefore, $\Theta_{a, \ell}(z)$ is a modular form of weight 2 on $\Gamma_{0}(\operatorname{lcm}[\ell,(4 a-1)])$.

Let $(a, \ell)$ be an element of $\mathbf{A}$. Consider the quadratic form $\mathcal{Q}_{a, \ell}$. By the above lemma, the corresponding theta series $\Theta_{a, \ell}(z)$ is a modular form in the space $M_{2}\left(\Gamma_{0}(\operatorname{lcm}[\ell,(4 a-1)])\right)$. Let 
us assume that the dimension of this vector space is $d_{a, \ell}$. If $\left\{f_{i}: 1 \leq i \leq d_{a, \ell}\right\}$ is a basis of $M_{2}\left(\Gamma_{0}(\operatorname{lcm}[\ell,(4 a-1)])\right)$, then we can write the theta series $\Theta_{a, \ell}(z)$ in terms of this basis. So, let

$$
\Theta_{a, \ell}(z)=\sum_{i=1}^{d_{a, \ell}} c_{i} f_{i}(z) .
$$

Combining this with (34) and comparing the $n$-th Fourier coefficients, we obtain the required formulas for $R_{a, \ell}(n)$.

We shall give below a basis of the modular forms space used in our formulas corresponding to each pair $(a, \ell)$ in the set $\mathbf{A}$. Using these bases, the formulas mentioned in Theorem 2.1 follow by comparing the $n$-th Fourier coefficients as demonstrated above. We shall be using the notation (7) for the eta-quotients.

Before we proceed, we define certain modular form of weight 2 using the quasimodular form $E_{2}(z)$. For natural numbers $a, b$ with $a \mid b, a \neq b$, define the function $\Phi_{a, b}(z)$ by

$$
\Phi_{a, b}(z)=\frac{1}{b-a}\left(b E_{2}(b z)-a E_{2}(a z)\right) .
$$

Using the transformation properties of $E_{2}(z)$, it follows that $\Phi_{a, b}(z)$ is a modular form belonging to the space $M_{2}\left(\Gamma_{0}(b)\right)$. We shall use these type of forms to construct our bases for the spaces of modular forms of weight 2 .

A basis for the space $M_{2}\left(\Gamma_{0}(6)\right)$ (the case $\left.(a, \ell)=(1,2)\right)$ : The space $M_{2}\left(\Gamma_{0}(6)\right)$ is 3 dimensional and a basis is given by

$$
\left\{\Phi_{1,2}(z), \Phi_{1,3}(z), \Phi_{1,6}(z)\right\}
$$

Therefore,

$$
\begin{aligned}
\Theta_{1,2}(z) & =\frac{1}{4} \Phi_{1,2}(z)-\frac{1}{2} \Phi_{1,3}(z)+\frac{5}{4} \Phi_{1,6}(z) \\
& =-\frac{1}{4} E_{2}(z)+\frac{1}{2} E_{2}(2 z)-\frac{3}{4} E_{2}(3 z)+\frac{3}{2} E_{2}(6 z),
\end{aligned}
$$

from which the formula for $R_{1,2}(n)$ follows.

A basis for the space $M_{2}\left(\Gamma_{0}(9)\right)$ (the case $(a, \ell)=(1,3)$ ): The vector space $M_{2}\left(\Gamma_{0}(9)\right)$ has dimension 3 and we use the following basis.

$$
\left\{\Phi_{1,3}(z), \Phi_{1,9}(z), \Psi_{2,9}(z)\right\}
$$

where $\Psi_{2,9}(z)$ is the eta-quotient $\Psi_{2,9}(z)=\frac{\eta^{3}(z) \eta^{3}(9 z)}{\eta^{2}(3 z)}$. We have

$$
\begin{aligned}
\Theta_{1,3}(z) & =\Phi_{1,9}(z)+3 \Psi_{2,9}(z) \\
& =-\frac{1}{8} E_{2}(z)+\frac{9}{8} E_{2}(9 z)+3 \Psi_{2,9}(z) .
\end{aligned}
$$

A basis for the space $M_{2}\left(\Gamma_{0}(12)\right)$ (the case $\left.(a, \ell)=(1,4)\right)$ : A basis for the space $M_{2}\left(\Gamma_{0}(12)\right)$ (which has dimension 5) is given by

$$
\left\{\Phi_{1,2}(z), \Phi_{1,3}(z), \Phi_{1,4}(z), \Phi_{1,6}(z), \Phi_{1,12}(z)\right\} .
$$

So, $\Theta_{1,4}(z)$ can be written as

$$
\begin{aligned}
\Theta_{1,4}(z) & =\frac{3}{8} \Phi_{1,2}(z)+\frac{1}{2} \Phi_{1,3}(z)-\frac{3}{4} \Phi_{1,4}(z)-\frac{15}{8} \Phi_{1,6}(z)+\frac{11}{4} \Phi_{1,12}(z) \\
& =-\frac{1}{4} E_{2}(z)+\frac{3}{4} E_{2}(2 z)+\frac{3}{4} E_{2}(3 z)-E_{2}(4 z)-\frac{9}{4} E_{2}(6 z)+3 E_{2}(12 z) .
\end{aligned}
$$


A basis for the space $M_{2}\left(\Gamma_{0}(15)\right)$ (the case $\left.(a, \ell)=(1,5)\right)$ : The vector space $M_{2}\left(\Gamma_{0}(15)\right)$ has dimension 4 and the subspace of cusp forms $S_{2}\left(\Gamma_{0}(15)\right)$ is one dimensional. Let $\Delta_{2,15}(z)$ be the unique normalized newform in the space $S_{2}\left(\Gamma_{0}(15)\right)$, which is given by an eta-quotient and we put

$$
\Delta_{2,15}(z)=1^{1} 3^{1} 5^{1} 15^{1}=\sum_{n \geq 1} \tau_{2,15}(n) q^{n} .
$$

We consider the following basis for $M_{2}\left(\Gamma_{0}(15)\right)$ :

$$
\left\{\Phi_{1,3}(z), \Phi_{1,5}(z), \Phi_{1,15}(z), \Delta_{2,15}(z)\right\} .
$$

In this case, we have

$$
\begin{aligned}
\Theta_{1,5}(z) & =-\frac{1}{8} \Phi_{1,3}(z)+\frac{1}{4} \Phi_{1,5}(z)+\frac{7}{8} \Phi_{1,15}(z)+\frac{9}{2} \Delta_{2,15}(z) \\
& =-\frac{1}{16} E_{2}(z)-\frac{3}{16} E_{2}(3 z)+\frac{5}{16} E_{2}(5 z)+\frac{15}{16} E_{2}(15 z)+\frac{9}{2} \Delta_{2,15}(z) .
\end{aligned}
$$

A basis for the space $M_{2}\left(\Gamma_{0}(14)\right)$ (the case $\left.(a, \ell)=(2,2)\right)$ : A basis for the 4 dimensional vector space $M_{2}\left(\Gamma_{0}(14)\right)$ is given by

$$
\left\{\Phi_{1,2}(z), \Phi_{1,7}(z), \Phi_{1,14}(z), \Delta_{2,14}(z)\right\},
$$

where $\Delta_{2,14}(z)$ is the unique normalized newform in $S_{2}\left(\Gamma_{0}(14)\right)$, which is given by

$$
\Delta_{2,14}(z)=1^{1} 2^{1} 7^{1} 14^{1}=\sum_{n \geq 1} \tau_{2,14}(n) q^{n} .
$$

With this basis, the theta series $\Theta_{2,2}(z)$ has the following expression.

$$
\begin{aligned}
\Theta_{2,2}(z) & =-\frac{1}{18} \Phi_{1,2}(z)+\frac{1}{3} \Phi_{1,7}(z)+\frac{13}{18} \Phi_{1,14}(z)+\frac{2}{3} \Delta_{2,14}(z) \\
& =-\frac{1}{18} E_{2}(z)-\frac{1}{9} E_{2}(2 z)+\frac{7}{18} E_{2}(7 z)+\frac{7}{9} E_{2}(14 z)+\frac{2}{3} \Delta_{2,14}(z) .
\end{aligned}
$$

A basis for the space $M_{2}\left(\Gamma_{0}(21)\right)$ (the case $\left.(a, \ell)=(2,3)\right)$ : Let $\Delta_{2,21}(z)$ be the unique normalized newform in $S_{2}\left(\Gamma_{0}(21)\right)$, which is given by the following eta-quotient:

$$
\begin{aligned}
\Delta_{2,21}(z)= & \frac{\eta(7 z)}{2 \eta^{2}(z) \eta(3 z) \eta(9 z) \eta(21 z)}\left(3 \eta^{2}(z) \eta^{2}(7 z) \eta^{4}(9 z)-\eta^{5}(3 z) \eta(7 z) \eta(9 z) \eta(21 z)+3 \eta^{4}(z) \eta^{2}(9 z) \eta^{2}(63 z)\right. \\
& \left.+7 \eta(z) \eta^{2}(3 z) \eta(9 z) \eta^{4}(21 z)+3 \eta^{3}(z) \eta(7 z) \eta^{3}(9 z) \eta(63 z)-3 \eta(z) \eta^{5}(3 z) \eta(21 z) \eta(63 z)\right)
\end{aligned}
$$

Now a basis for this space is given by

$$
\left\{\Phi_{1,3}(z), \Phi_{1,7}(z), \Phi_{1,21}(z), \Delta_{2,21}(z)\right\} .
$$

We give the expression for the corresponding theta series.

$$
\begin{aligned}
\Theta_{2,3}(z) & =\frac{1}{8} \Phi_{1,3}(z)-\frac{3}{8} \Phi_{1,7}(z)+\frac{21}{16} \Phi_{1,21}(z)+\frac{1}{2} \Delta_{2,21}(z) \\
& =-\frac{21}{320} E_{2}(z)+\frac{3}{16} E_{2}(3 z)-\frac{7}{16} E_{2}(7 z)+\frac{441}{320} E_{2}(21 z)+\frac{1}{2} \Delta_{2,21}(z) .
\end{aligned}
$$

A basis for the space $M_{2}\left(\Gamma_{0}(28)\right)$ (the case $\left.(a, \ell)=(2,4)\right)$ : In this case, the cusp forms space $S_{2}\left(\Gamma_{0}(28)\right)$ is spanned by $\Delta_{2,14}(z)$ and $\Delta_{2,14}(2 z)$ and we use the following basis:

$$
\left\{\Phi_{1,2}(z), \Phi_{1,4}(z), \Phi_{1,7}(z), \Phi_{1,14}(z), \Phi_{1,28}(z), \Delta_{2,14}(z), \Delta_{2,14}(2 z)\right\} .
$$


The newform $\Delta_{2,14}(z)$ is given by (37). We give the expression for the theta series.

$$
\begin{aligned}
\Theta_{2,4}(z)= & -\frac{1}{72} \Phi_{1,2}(z)-\frac{1}{12} \Phi_{1,4}(z)+\frac{1}{6} \Phi_{1,7}(z)+\frac{13}{72} \Phi_{1,14}(z)+\frac{3}{4} \Phi_{1,28}(z)+\frac{4}{3} \Delta_{2,14}(z)+\frac{8}{3} \Delta_{2,14}(2 z) \\
= & -\frac{1}{36} E_{2}(z)-\frac{1}{36} E_{2}(2 z)-\frac{1}{9} E_{2}(4 z)+\frac{7}{36} E_{2}(7 z)+\frac{7}{36} E_{2}(14 z)+\frac{7}{9} E_{2}(28 z) \\
& +\frac{4}{3} \Delta_{2,14}(z)+\frac{8}{3} \Delta_{2,14}(2 z) .
\end{aligned}
$$

A basis for the space $M_{2}\left(\Gamma_{0}(22)\right)$ (the case $\left.(a, \ell)=(3,2)\right)$ : First we give the newform of weight 2 on $\Gamma_{0}(11)$.

$$
\Delta_{2,11}(z)=1^{2} 11^{2}=\sum_{n \geq 1} \tau_{2,11}(n) q^{n}
$$

For getting the required formula, we use the following basis:

$$
\left\{\Phi_{1,2}(z), \Phi_{1,11}(z), \Phi_{1,22}(z), \Delta_{2,11}(z), \Delta_{2,11}(2 z)\right\} .
$$

The expression for the theta series $\Theta_{3,2}(z)$ is given below.

$$
\begin{aligned}
\Theta_{3,2}(z) & =\frac{1}{12} \Phi_{1,2}(z)-\frac{5}{6} \Phi_{1,11}(z)+\frac{7}{4} \Phi_{1,22}(z) \\
& =-\frac{1}{12} E_{2}(z)+\frac{1}{6} E_{2}(2 z)-\frac{11}{12} E_{2}(11 z)+\frac{11}{6} E_{2}(22 z) .
\end{aligned}
$$

A basis for the space $M_{2}\left(\Gamma_{0}(33)\right)$ (the case $\left.(a, \ell)=(3,3)\right)$ : In this case the dimension of the space is 6 . We need the newform of level 33. Since explicit expression of this newform is not known, we give below its first few Fourier coefficients (using SAGE).

$$
\Delta_{2,33}(z)=q+q^{2}-q^{3}-q^{4}-2 q^{5}-q^{6}+4 q^{7}-3 q^{8}+q^{9}-2 q^{10}+O\left(q^{11}\right)
$$

We use the following basis for $M_{2}\left(\Gamma_{0}(33)\right)$ :

$$
\left\{\Phi_{1,3}(z), \Phi_{1,11}(z), \Phi_{1,33}(z), \Delta_{2,11}(z), \Delta_{2,11}(3 z), \Delta_{2,33}(z)\right\} .
$$

Using this basis, we have

$$
\begin{aligned}
\Theta_{3,3}(z) & =-\frac{1}{20} \Phi_{1,3}(z)+\frac{1}{4} \Phi_{1,11}(z)+\frac{4}{5} \Phi_{1,33}(z)+\frac{16}{15} \Delta_{2,11}(z)+\frac{16}{5} \Delta_{2,11}(3 z)+\frac{1}{3} \Delta_{2,33}(z) \\
& =-\frac{1}{40} E_{2}(z)-\frac{3}{40} E_{2}(3 z)+\frac{11}{40} E_{2}(11 z)+\frac{33}{40} E_{2}(33 z)+\frac{16}{15} \Delta_{2,11}(z)+\frac{16}{5} \Delta_{2,11}(3 z)+\frac{1}{3} \Delta_{2,33}(z) .
\end{aligned}
$$

A basis for the space $M_{2}\left(\Gamma_{0}(30)\right)$ (the case $\left.(a, \ell)=(4,2)\right)$ : The normalized newform of level 15 is given by (36). For level 30 it is defined below.

$$
\Delta_{2,30}(z)=3^{1} 5^{1} 6^{1} 10^{1}-1^{1} 2^{1} 15^{1} 30^{1}=\sum_{n \geq 1} \tau_{2,30}(n) q^{n} .
$$

Following is a basis for the space $M_{2}\left(\Gamma_{0}(30)\right)$.

$$
\left\{\Phi_{1,2}(z), \Phi_{1,3}(z), \Phi_{1,5}(z), \Phi_{1,6}(z), \Phi_{1,10}(z), \Phi_{1,15}(z), \Phi_{1,30}(z), \Delta_{2,15}(z), \Delta_{2,15}(2 z), \Delta_{2,30}(z)\right\} .
$$

Using the above basis, we have 


$$
\begin{aligned}
\Theta_{4,2}(z)= & -\frac{1}{48} \Phi_{1,2}(z)-\frac{1}{24} \Phi_{1,3}(z)+\frac{1}{12} \Phi_{1,5}(z)-\frac{5}{48} \Phi_{1,6}(z)+\frac{3}{16} \Phi_{1,10}(z)+\frac{7}{24} \Phi_{1,15}(z) \\
& +\frac{29}{48} \Phi_{1,30}(z)+\frac{1}{2} \Delta_{2,15}(z)+\Delta_{2,15}(2 z)+\Delta_{2,30}(z) \\
= & -\frac{1}{48} E_{2}(z)-\frac{1}{24} E_{2}(2 z)-\frac{1}{16} E_{2}(3 z)+\frac{5}{48} E_{2}(5 z)-\frac{1}{8} E_{2}(6 z)+\frac{5}{24} E_{2}(10 z)+\frac{5}{16} E_{2}(15 z) \\
& +\frac{5}{8} E_{2}(30 z)+\frac{1}{2} \Delta_{2,15}(z)+\Delta_{2,15}(2 z)+\Delta_{2,30}(z) .
\end{aligned}
$$

A basis for the space $M_{2}\left(\Gamma_{0}(19)\right)$ (the case $(a, \ell)=(5,1)$ ): For defining the newform of level 19 , we use the Ramanujan theta functions $\Phi(z)$ and $\Psi(z)$ which are defined below.

$$
\begin{aligned}
& \Phi(z):=\frac{\eta^{5}(2 z)}{\eta^{2}(z) \eta^{2}(4 z)}, \\
& \Psi(z):=q^{-1 / 8} \frac{\eta^{2}(2 z)}{\eta(z)} .
\end{aligned}
$$

We give the newform $\Delta_{2,19}(z)$ as follows.

$$
\Delta_{2,19}(z)=q\left\{\Psi(4 z) \Phi(38 z)-q^{2} \Psi(z) \Psi(19 z)+q^{9} \Phi(2 z) \Psi(76 z)\right\}^{2}:=\sum_{n \geq 1} \tau_{2,19}(n) q^{n}
$$

The vector space $M_{2}\left(\Gamma_{0}(19)\right)$ is spanned by the following two modular forms:

$$
\left\{\Phi_{1,19}(z), \Delta_{2,19}(z)\right\}
$$

Now we give the expression for the corresponding theta function.

$$
\begin{aligned}
\Theta_{5,1}(z) & =\Phi_{1,19}(z)+\frac{8}{3} \Delta_{2,19}(z) \\
& =-\frac{1}{18} E_{2}(z)+\frac{19}{18} E_{2}(19 z)+\frac{8}{3} \Delta_{2,19}(z) .
\end{aligned}
$$

A basis for the space $M_{2}\left(\Gamma_{0}(38)\right)$ (the case $(a, \ell)=(5,2)$ ): In this case we need two newforms of level 38. Explicit expression of these newforms are not known. However, using SAGE one can get their Fourier expansion (with certain number of Fourier coefficients) which we give below.

$$
\begin{aligned}
& \Delta_{2,38 ; 1}(z)=q-q^{2}+q^{3}+q^{4}-q^{6}-q^{7}-q^{8}-2 q^{9}+O\left(q^{10}\right)=\sum_{n \geq 1} \tau_{2,38 ; 1}(n) q^{n} \\
& \Delta_{2,38 ; 2}(z)=q+q^{2}-q^{3}+q^{4}-4 q^{5}-q^{6}+3 q^{7}+q^{8}-2 q^{9}+O\left(q^{10}\right)=\sum_{n \geq 1} \tau_{2,38 ; 2}(n) q^{n} .
\end{aligned}
$$

A basis for the space $M_{2}\left(\Gamma_{0}(38)\right)$ is given by

$$
\left\{\Phi_{1,2}(z), \Phi_{1,19}(z), \Phi_{1,5}(z), \Phi_{1,38}(z), \Delta_{2,19}(z), \Delta_{2,19}(2 z), \Delta_{2,38 ; 1}(z), \Delta_{2,38 ; 2}(z)\right\} .
$$

In this case, the theta series has the following expression.

$$
\begin{aligned}
\Theta_{5,2}(z) & =\frac{1}{20} \Phi_{1,2}(z)-\frac{9}{10} \Phi_{1,19}(z)+\frac{37}{20} \Phi_{1,5}(z)+\frac{4}{5} \Delta_{2,38 ; 2}(z) \\
& =-\frac{1}{20} E_{2}(z)+\frac{1}{10} E_{2}(2 z)-\frac{19}{20} E_{2}(19 z)+\frac{19}{10} E_{2}(38 z)+\frac{4}{5} \Delta_{2,38 ; 2}(z) .
\end{aligned}
$$

Proof of Theorem 2.1 is now complete. 
4.2. Proof of Theorem 2.2. We shall demonstrate the method by giving a proof of the formula for $R_{1,2 ; j}(n), 1 \leq j \leq 4$. The rest of the proofs are similar. It is clear that

$$
R_{1,2 ; j}(n)=\sum_{\substack{a, b \in \mathbb{N}_{0} \\ a+b j=n}} R_{1,2}(a) R_{1,2}(b) .
$$

Now using the formula for $R_{1,2}(n)$ from Theorem 2.1 with the convention $R_{1,2}(0)=1$, we get

$$
\begin{aligned}
R_{1,2 ; j}(n)= & R_{1,2}(n)+R_{1,2}(n / j)+\sum_{\substack{a, b \in \mathbb{N} \\
a+b j=n}} R_{1,2}(a) R_{1,2}(b) \\
= & R_{1,2}(n)+R_{1,2}(n / j)+\sum_{\substack{a, b \in \mathbb{N} \\
a+b j=n}}(6 \sigma(a)-12 \sigma(a / 2)+18 \sigma(a / 3)-36 \sigma(a / 6)) \\
= & R_{1,2}(n)+R_{1,2}(n / j)+36 W_{j}(n)-72 W_{2 j}(n)+108 W_{3 j}(n)-216 W_{6 j}(n)-72 W_{2, j}(n) \\
& +108 W_{3, j}(n)-216 W_{6, j}(n)-216 W_{2,3 j}(n)-216 W_{3,2 j}(n)+144 W_{j}(n / 2)+324 W_{j}(n / 3) \\
& +1296 W_{j}(n / 6)-648 W_{2 j}(n / 3)+432 W_{3 j}(n / 2)-648 W_{2, j}(n / 3)+432 W_{3, j}(n / 2) .
\end{aligned}
$$

\begin{tabular}{|c|c|c|c|}
\hline$(a, \ell ; j)$ & $\begin{array}{c}\text { Convolution sums } \\
W_{N}(n)\end{array}$ & $\begin{array}{c}\text { Convolution sums } \\
W_{a, b}(n)\end{array}$ & References \\
\hline$\overline{(1,2 ; 1)}$ & $W_{N}(n), N=1,2,3,6$ & $W_{2,3}(n)$ & [5, 18 \\
\hline$(1,2 ; 2)$ & $W_{N}(n), N=1,2,3,4,6,12$ & $W_{2,3}(n), W_{3,4}(n)$ & [5, 16, 18, \\
\hline$(1,2 ; 3)$ & $W_{N}(n), N=1,2,3,6,9,18$ & $W_{2,3}(n), W_{2,9}(n)$ & $1,5,18$ \\
\hline$\overline{(1,2 ; 4)}$ & $W_{N}(n), N=2,4,6,8,12,24$ & $W_{2,3}(n), W_{3,4}(n), W_{3,8}(n)$ & {$[2,5,16,18$} \\
\hline$(1,4 ; 1)$ & $W_{N}(n), N=1,2,3,4,6,12$ & $W_{2,3}(n), W_{3,4}(n)$ & $5,16,18$ \\
\hline$(1,4 ; 2)$ & $W_{N}(n), N=1,2,4,6,8,12,24$ & $W_{2,3}(n), W_{3,4}(n), W_{3,8}(n)$ & {$[2,5,16,18$} \\
\hline$\overline{(3,2 ; 1)}$ & $W_{N}(n), N=1,2,22$ & $W_{2,11}(n)$ & {$[15,[18$} \\
\hline
\end{tabular}

We now use the convolution sums $W_{a, b}(n)$ and $W_{N}(n)$ obtained by several authors (see the table below) in the last step and get the required formulas for $R_{1,2 ; j}(n)$ for $1 \leq j \leq 4$.

To get the formula for $R_{3,2 ; 1}(n)$ we also need the convolution sum $W_{11}(n)$. Though this convolution sum is obtained by E. Royer in [18], it involved a pair of terms with complex coefficients. In order to avoid this expression, we compute below the convolution sum $W_{11}(n)$ which involves only the rational coefficients.

The convolution sum $W_{11}(n)$ : First we compute an explicit basis for the space $M_{4}\left(\Gamma_{0}(22)\right)$. The dimension of this vector space is 11 and the cuspidal dimension is 7 . The following 7 eta-quotients form a basis for the space of cusp forms $S_{4}\left(\Gamma_{0}(22)\right)$.

$$
\begin{aligned}
& A_{1}(z)=1^{6} 2^{-2} 11^{6} 22^{-2}:=\sum_{n \geq 1} a_{1}(n) q^{n} \\
& A_{2}(z)=1^{4} 11^{4}:=\sum_{n \geq 1} a_{2}(n) q^{n} \\
& A_{3}(z)=1^{2} 2^{2} 11^{2} 22^{2}:=\sum_{n \geq 1} a_{3}(n) q^{n}
\end{aligned}
$$




$$
\begin{aligned}
& A_{4}(z)=2^{4} 22^{4}:=\sum_{n \geq 1} a_{4}(n) q^{n}, \\
& A_{5}(z)=1^{-2} 2^{6} 11^{-2} 22^{6}:=\sum_{n \geq 1} a_{5}(n) q^{n}, \\
& A_{6}(z)=1^{-1} 2^{1} 11^{3} 22^{5}:=\sum_{n \geq 1} a_{6}(n) q^{n}, \\
& A_{7}(z)=1^{-5} 2^{9} 11^{7} 22^{-3}:=\sum_{n \geq 1} a_{7}(n) q^{n} .
\end{aligned}
$$

By taking a basis of the Eisenstein series for the space $M_{4}\left(\Gamma_{0}(22)\right)$ as $\left\{E_{4}(t z): t \mid 22\right\}$, we get the following full basis for $M_{4}\left(\Gamma_{0}(22)\right)$.

$$
\left\{E_{4}(t z), A_{j}(z): t \mid 22,1 \leq j \leq 7\right\} .
$$

In order to get the convolution sum $W_{11}(n)$, we express the modular form of weight 4 given by $\left(E_{2}(z)-11 E_{2}(11 z)\right)^{2}$ in terms of the above basis. So, we get the following expression.

$$
\begin{aligned}
\left(E_{2}(z)-11 E_{2}(11 z)\right)^{2}= & \frac{50}{61} E_{4}(z)+\frac{6050}{61} E_{4}(11 z)+\frac{17280}{61} A_{1}(z)+\frac{118656}{61} A_{2}(z) \\
& +\frac{276480}{61} A_{3}(z)+\frac{276480}{61} A_{4}(z) .
\end{aligned}
$$

Now, by comparing the $n$-th coefficient on both the sides, we get the expression for $W_{11}(n)$ as

$$
\begin{aligned}
W_{11}(n)= & \frac{5}{1464} \sigma_{3}(n)+\frac{605}{1464} \sigma_{3}(n / 11)+\left(\frac{1}{21}-\frac{n}{44}\right) \sigma(n)+\left(\frac{1}{21}-\frac{n}{4}\right) \sigma(n / 11) \\
& -\frac{15}{671} a_{1}(n)-\frac{103}{671} a_{2}(n)-\frac{240}{671} a_{3}(n)-\frac{240}{671} a_{4}(n) .
\end{aligned}
$$

4.3. Proof of Theorem 2.3. We observe that the theta series corresponding to the quadratic form given by (4) is the following product:

$$
\Theta^{i}(z) \Theta^{j}(2 z) \Theta^{k}(4 z) \Theta^{l}(8 z) .
$$

Therefore, by Fact II of [17], all of them belong to the space of modular forms of weight 4 on $\Gamma_{0}(32)$ with character depending on the parity of $j+l$. When $j+l$ is even, then the above product of theta series belongs to $M_{4}\left(\Gamma_{0}(32)\right)$ and if $j+l$ is odd, then it belongs to $M_{4}\left(\Gamma_{0}(32), \chi_{8}\right)$. Therefore, as in the proof of Theorem 2.1, the essence of the proof lies in giving explicit bases for these vector spaces.

4.4. A basis for $M_{4}\left(\Gamma_{0}(32)\right)$ and proof of Theorem $\mathbf{2 . 3}(\mathbf{i})$. The vector space $M_{4}\left(\Gamma_{0}(32)\right)$ has dimension 16 and the space of Eisenstein series has dimension 8. So, $\operatorname{dim}_{\mathbb{C}} S_{4}\left(\Gamma_{0}(32)\right)=8$. For $d=8$ and $16, S_{4}^{\text {new }}\left(\Gamma_{0}(d)\right)$ is one-dimensional and $\operatorname{dim}_{\mathbb{C}} S_{4}^{\text {new }}\left(\Gamma_{0}(32)\right)=3$.

Let us define some eta-quotients and use them to give an explicit basis for $S_{4}\left(\Gamma_{0}(32)\right)$. Let

$$
\begin{gathered}
f_{4,8}(z)=2^{4} 4^{4}:=\sum_{n \geq 1} a_{4,8}(n) q^{n}, \\
f_{4,16}(z)=2^{-4} 4^{16} 8^{-4}:=\sum_{n \geq 1} a_{4,16}(n) q^{n},
\end{gathered}
$$




$$
\begin{gathered}
g_{4,32,1}(z)=1^{-2} 2^{1} 4^{8} 8^{3} 16^{-2}:=\sum_{n \geq 1} a_{4,32,1}(n) q^{n}, \\
g_{4,32,2}(z)=1^{2} 2^{3} 8^{1} 16^{2}:=\sum_{n \geq 1} a_{4,32,2}(n) q^{n}, \\
g_{4,32,3}(z)=1^{-4} 2^{6} 4^{8} 8^{-2}:=\sum_{n \geq 1} a_{4,32,3}(n) q^{n}, \\
f_{4,32,1}(z):=\sum_{n \equiv 1(2)} a_{4,32,1}(n) q^{n}, \\
f_{4,32,2}(z):=\sum_{n \equiv 1(2)} a_{4,32,2}(n) q^{n}, \\
f_{4,32,3}(z):=\sum_{n \equiv 1(2)} a_{4,32,3}(n) q^{n} .
\end{gathered}
$$

Let $\chi_{-4}$ be the primitive odd character modulo 4. Using the definition (8), the Eisenstein series $E_{4, \chi_{-4}, \chi_{-4}}(z)$ belongs to $M_{4}\left(\Gamma_{0}(16)\right)$ and we have

$$
E_{4, \chi_{-4}, \chi_{-4}}(z)=\sum_{n \geq 1} \sigma_{3, \chi_{-4}, \chi_{-4}}(n) q^{n}=\sum_{n \geq 1}\left(\frac{-4}{n}\right) \sigma_{3}(n) q^{n} .
$$

Using the above functions, we give below a basis for the space $M_{4}\left(\Gamma_{0}(32)\right)$.

Proposition 4.2. A basis for the space $M_{4}\left(\Gamma_{0}(32)\right)$ is given by

$$
\begin{aligned}
& \left\{E_{4}(t z), t \mid 32 ; E_{4, \chi_{-4}, \chi_{-4}}(z), E_{4, \chi_{-4}, \chi_{-4}}(2 z),\right. \\
& \left.\quad f_{4,8}\left(t_{1} z\right), t_{1}\left|4 ; f_{4,16}\left(t_{2} z\right), t_{2}\right| 2 ; f_{4,32,1}(z), f_{4,32,2}(z), f_{4,32,3}(z)\right\} .
\end{aligned}
$$

For the sake of simplicity in the formulae, we list these basis elements as $\left\{F_{\alpha}(z) \mid 1 \leq \alpha \leq 16\right\}$, where $F_{1}(z)=E_{4}(z), F_{2}(z)=E_{4}(2 z), F_{3}(z)=E_{4}(4 z), F_{4}(z)=E_{4}(8 z), F_{5}(z)=E_{4}(16 z), F_{6}(z)=E_{4}(32 z)$, $F_{7}(z)=E_{4, \chi_{-4}, \chi_{-4}}(z), F_{8}(z)=E_{4, \chi_{-4}, \chi_{-4}}(2 z), F_{9}(z)=f_{4,8}(z), F_{10}(z)=f_{4,8}(2 z), F_{11}(z)=f_{4,8}(4 z)$, $F_{12}(z)=f_{4,16}(z), F_{13}(z)=f_{4,16}(2 z), F_{14}(z)=f_{4,32,1}(z), F_{15}(z)=f_{4,32,2}(z), F_{16}(z)=f_{4,32,3}(z)$.

We also express the Fourier coefficients of the function $F_{\alpha}(z)=\sum_{n \geq 1} C_{\alpha}(n) q^{n}, 1 \leq \alpha \leq 16$.

We are now ready to prove the theorem. Noting that all the 40 cases (corresponding to Type I in Table 2) have the property that the sum of the powers of the theta functions corresponding to the coefficients 2 and 8 are even. So, we can express these theta functions as a linear combination of the basis given in Proposition 4.2 as follows.

$$
\Theta^{i}(z) \Theta^{j}(2 z) \Theta^{k}(4 z) \Theta^{l}(8 z)=\sum_{\alpha=1}^{16} c_{\alpha} F_{\alpha}(z)
$$

where $a_{\alpha}$ 's some constants. Comparing the $n$-th Fourier coefficients both the sides, we get

$$
N\left(1^{i}, 2^{j}, 4^{k}, 8^{l} ; n\right)=\sum_{\alpha=1}^{16} c_{\alpha} C_{\alpha}(n) .
$$

Explicit values for the constants $c_{\alpha}, 1 \leq \alpha \leq 16$ corresponding to these 40 cases are given in Table 3 .

4.5. A basis for $M_{4}\left(\Gamma_{0}(32), \chi_{8}\right)$ and proof of Theorem 2.3 (ii). The space $M_{4}\left(\Gamma_{0}(32), \chi_{8}\right)$ is 16 dimensional and the cusp forms space has dimension 8. For the space of Eisenstein series we use the basis elements given by (8). There are two Eisenstein series corresponding to $(\chi, \psi)=\left(\mathbf{1}, \chi_{8}\right)$ 
and $(\chi, \psi)=\left(\chi_{8}, \mathbf{1}\right)$, where $\chi_{8}=\left(\frac{2}{2}\right)$, the even primitive character modulo 8 . For the space of cusp forms, we use the following two newforms of level 8 .

$$
\begin{aligned}
& f_{4,8, \chi_{8} ; 1}(z)=1^{-2} 2^{11} 4^{-3} 8^{2}:=\sum_{n \geq 1} a_{4,8, \chi_{8} ; 1}(n) q^{n}, \\
& f_{4,8, \chi_{8} ; 2}(z)=1^{2} 2^{-3} 4^{11} 8^{-2}:=\sum_{n \geq 1} a_{4,8, \chi_{8} ; 2}(n) q^{n},
\end{aligned}
$$

We also need the 2 newforms of level 32, which we define below. Let

$$
\begin{aligned}
& g_{4,32, \chi_{8} ; 1}(z)=1^{2} 2^{1} 4^{5}:=\sum_{n \geq 1} a_{4,32, \chi_{8} ; 1}(n) q^{n}, \\
& g_{4,32, \chi_{8} ; 2}(z)=1^{-2} 2^{3} 4^{3} 8^{4}:=\sum_{n \geq 1} a_{4,32, \chi_{8} ; 2}(n) q^{n} .
\end{aligned}
$$

Then the two newforms of level 32 are defined by

$$
\begin{aligned}
f_{4,32, \chi_{8} ; 1}(z) & :=\sum_{n \geq 1} \chi_{4}(n) a_{4,32, \chi_{8} ; 1}(n) q^{n}, \\
f_{4,32, \chi_{8} ; 2}(z) & :=\sum_{n \geq 1} \chi_{4}(n) a_{4,32, \chi_{8} ; 2}(n) q^{n},
\end{aligned}
$$

where $\chi_{4}$ is the trivial character modulo 4 .

A basis for the space $M_{4}\left(\Gamma_{0}(32), \chi_{8}\right)$ is given in the following proposition.

Proposition 4.3. A basis for the space $M_{4}\left(\Gamma_{0}(32), \chi_{8}\right)$ is given by

$$
\begin{aligned}
& \left\{E_{4, \mathbf{1}, \chi_{8}}(t z), E_{4, \chi_{8}, \mathbf{1}}(t z), t \mid 4 ; E_{4, \chi_{-4}, \chi_{-8}}(z), E_{4, \chi_{-8}, \chi_{-4}}(z)\right. \\
& \left.f_{4,8, \chi_{8} ; 1}\left(t_{1} z\right), f_{4,8, \chi_{8} ; 2}\left(t_{1} z\right), t_{1} \mid 4 ; f_{4,32, \chi_{8} ; 1}(z), f_{4,32, \chi_{8} ; 2}(z)\right\} .
\end{aligned}
$$

In the above, $E_{4, \mathbf{1}, \chi_{8}}(z)$ and $E_{4, \chi_{8}, \mathbf{1}}(z)$ are defined as in (8) $, f_{4,8, \chi_{8} ; i}(z), i=1,2$ are defined in (64) and $f_{4,32, \chi_{8} ; j}(z), 1 \leq j \leq 2$ are defined by (66)

For the sake of simplifying of the notation, we shall list the basis in Proposition 4.3 as $\left\{G_{\alpha}(z) \mid 1 \leq \alpha \leq 16\right\}$, where $G_{1}(z)=E_{4, \mathbf{1}, \chi_{8}}(z), G_{2}(z)=E_{4, \mathbf{1}, \chi_{8}}(2 z), G_{3}(z)=E_{4, \mathbf{1}, \chi_{8}}(4 z), G_{4}(z)=$ $E_{4, \chi_{8}, \mathbf{1}}(z), G_{5}(z)=E_{4, \chi_{8}, \mathbf{1}}(2 z), G_{6}(z)=E_{4, \chi_{8}, \mathbf{1}}(4 z), G_{7}(z)=E_{4, \chi_{-4}, \chi_{-8}}(z), G_{8}(z)=E_{4, \chi_{-8}, \chi_{-4}}(z)$, $G_{9}(z)=f_{4,8, \chi_{8} ; 1}(z), G_{10}(z)=f_{4,8, \chi_{8} ; 1}(2 z), G_{11}(z)=f_{4,8, \chi_{8} ; 1}(4 z), G_{12}(z)=f_{4,8, \chi_{8} ; 2}(z), G_{13}(z)=$ $f_{4,8, \chi_{8} ; 2}(2 z), G_{14}(z)=f_{4,8, \chi_{8} ; 2}(4 z), G_{15}(z)=f_{4,32, \chi_{8} ; 1}(z), G_{16}(z)=f_{4,32, \chi_{8} ; 2}(z)$.

As before, we also write the Fourier expansions of these basis elements as $G_{\alpha}(z)=\sum_{n \geq 1} D_{\alpha}(n) q^{n}$, $1 \leq \alpha \leq 16$.

In this case, all the 44 quadruples (corresponding to Type II in Table 2) have the property that the sum of the powers of the theta functions corresponding to the coefficients 2 and 8 are odd. Therefore, the resulting products of theta functions are modular forms of weight 4 on $\Gamma_{0}(32)$ with character $\chi_{8}$ (as observed earlier). So, we can express these products of theta functions as a linear combination of the basis given in Proposition 4.3 as follows.

$$
\Theta^{i}(z) \Theta^{j}(2 z) \Theta^{k}(3 z) \Theta^{l}(4 z)=\sum_{\alpha=1}^{16} d_{\alpha} G_{\alpha}(z) .
$$

Comparing the $n$-th Fourier coefficients both the sides, we get

$$
N\left(1^{i}, 2^{j}, 4^{k}, 8^{l} ; n\right)=\sum_{\alpha=1}^{16} d_{\alpha} D_{\alpha}(n) .
$$

Explicit values for the constants $d_{\alpha}, 1 \leq \alpha \leq 14$ corresponding to these 44 cases are given in Table 4. 


\section{List OF TABLES}

In this section, we list Tables 3 and 4 , which give the coefficients for the formulas for the number of representations corresponding to Theorem 2.3 (i) and (ii).

Table 3. (Theorem 2.3 (i))

\begin{tabular}{|c|c|c|c|c|c|c|c|c|c|c|c|c|c|c|c|c|}
\hline$i j k l$ & $c_{1}$ & $c_{2}$ & $c_{3}$ & $c_{4}$ & $c_{5}$ & $c_{6}$ & $c_{7}$ & $c_{8}$ & $c_{9}$ & $c_{10}$ & $c_{11}$ & $c_{12}$ & $c_{13}$ & $c_{14}$ & $c_{15}$ & $c_{16}$ \\
\hline 1016 & $\frac{1}{15360}$ & $\frac{-3}{5120}$ & $\frac{17}{1920}$ & $\frac{-1}{120}$ & $\frac{-1}{15}$ & $\frac{16}{15}$ & $\frac{1}{64}$ & 0 & $\frac{31}{64}$ & 0 & 2 & $\frac{31}{64}$ & 0 & $\frac{13}{8}$ & $\frac{3}{4}$ & $\frac{-5}{8}$ \\
\hline 1034 & $\frac{1}{7680}$ & $\frac{-3}{2560}$ & $\frac{17}{960}$ & $\frac{-1}{60}$ & $\frac{-1}{15}$ & $\frac{16}{15}$ & $\frac{1}{32}$ & 0 & $\frac{15}{32}$ & 0 & 4 & $\frac{15}{32}$ & 0 & $\frac{3}{2}$ & 1 & $\frac{-1}{2}$ \\
\hline 1052 & $\frac{1}{3840}$ & $\frac{-3}{1280}$ & $\frac{17}{480}$ & $\frac{-1}{30}$ & $\frac{-1}{15}$ & $\frac{16}{15}$ & $\frac{1}{16}$ & 0 & $\frac{7}{16}$ & 0 & 4 & $\frac{7}{16}$ & 0 & $\frac{3}{2}$ & 1 & $\frac{-1}{2}$ \\
\hline 1115 & $\frac{1}{7680}$ & $\frac{-1}{7680}$ & 0 & 0 & $\frac{-1}{15}$ & $\frac{16}{15}$ & 0 & 0 & $\frac{11}{32}$ & $\frac{3}{4}$ & 2 & $\frac{5}{8}$ & 1 & $\frac{11}{8}$ & $\frac{1}{4}$ & $\frac{-3}{8}$ \\
\hline 1133 & $\frac{1}{3840}$ & $\frac{-1}{3840}$ & 0 & 0 & $\frac{-1}{15}$ & $\frac{16}{15}$ & 0 & 0 & $\frac{7}{16}$ & $\frac{1}{2}$ & 4 & $\frac{1}{2}$ & 1 & $\frac{5}{4}$ & $\frac{1}{2}$ & $\frac{-1}{4}$ \\
\hline 1151 & $\frac{1}{1920}$ & $\frac{-1}{1920}$ & 0 & 0 & $\frac{-1}{15}$ & $\frac{16}{15}$ & 0 & 0 & $\frac{3}{8}$ & 1 & 4 & $\frac{1}{2}$ & 0 & $\frac{3}{2}$ & 1 & $\frac{-1}{2}$ \\
\hline 1214 & $\frac{1}{3840}$ & $\frac{-1}{3840}$ & 0 & 0 & $\frac{-1}{15}$ & $\frac{16}{15}$ & 0 & 0 & $\frac{3}{16}$ & $\frac{3}{2}$ & 4 & $\frac{3}{4}$ & 2 & 1 & 0 & 0 \\
\hline 1232 & $\frac{1}{1920}$ & $\frac{-1}{1920}$ & 0 & 0 & $\frac{-1}{15}$ & $\frac{16}{15}$ & 0 & 0 & $\frac{3}{8}$ & 1 & 4 & $\frac{1}{2}$ & 2 & 1 & 0 & 0 \\
\hline 1313 & $\frac{1}{1920}$ & $\frac{-1}{1920}$ & 0 & 0 & $\frac{-1}{15}$ & $\frac{16}{15}$ & 0 & 0 & $\frac{1}{8}$ & 2 & 8 & $\frac{3}{4}$ & 3 & $\frac{1}{2}$ & 0 & $\frac{1}{2}$ \\
\hline 1331 & $\frac{1}{960}$ & $\frac{-1}{960}$ & 0 & 0 & $\frac{-1}{15}$ & $\frac{16}{15}$ & 0 & 0 & $\frac{1}{4}$ & 2 & 4 & $\frac{1}{2}$ & 2 & 1 & 0 & 0 \\
\hline 1412 & $\frac{1}{960}$ & $\frac{-1}{960}$ & 0 & 0 & $\frac{-1}{15}$ & $\frac{16}{15}$ & 0 & 0 & $\frac{1}{4}$ & 2 & 12 & $\frac{1}{2}$ & 4 & 0 & 0 & 1 \\
\hline 1511 & $\frac{1}{480}$ & $\frac{-1}{480}$ & 0 & 0 & $\frac{-1}{15}$ & $\frac{16}{15}$ & 0 & 0 & $\frac{1}{2}$ & 2 & 12 & 0 & 4 & 0 & 0 & 1 \\
\hline 2006 & $\frac{1}{7680}$ & $\frac{-1}{7680}$ & 0 & 0 & $\frac{-1}{15}$ & $\frac{16}{15}$ & $\frac{1}{32}$ & $\frac{1}{4}$ & $\frac{31}{32}$ & $\frac{7}{4}$ & 2 & $\frac{31}{32}$ & $\frac{7}{4}$ & $\frac{13}{4}$ & $\frac{3}{2}$ & $\frac{-5}{4}$ \\
\hline 2024 & $\frac{1}{3840}$ & $\frac{-1}{3840}$ & 0 & 0 & $\frac{-1}{15}$ & $\frac{16}{15}$ & $\frac{1}{16}$ & 0 & $\frac{15}{16}$ & $\frac{3}{2}$ & 4 & $\frac{15}{16}$ & 2 & 3 & 2 & -1 \\
\hline 2042 & $\frac{1}{1920}$ & $\frac{-1}{1920}$ & 0 & 0 & $\frac{-1}{15}$ & $\frac{16}{15}$ & $\frac{1}{8}$ & 0 & $\frac{7}{8}$ & 1 & 4 & $\frac{7}{8}$ & 2 & 3 & 2 & -1 \\
\hline 2105 & $\frac{1}{3840}$ & $\frac{-1}{3840}$ & $\frac{1}{120}$ & $\frac{-17}{120}$ & $\frac{1}{15}$ & $\frac{16}{15}$ & 0 & $\frac{1}{4}$ & $\frac{11}{16}$ & $\frac{5}{2}$ & 6 & $\frac{5}{4}$ & $\frac{11}{4}$ & $\frac{11}{4}$ & $\frac{1}{2}$ & $\frac{-3}{4}$ \\
\hline 2123 & $\frac{1}{1920}$ & $\frac{-1}{1920}$ & 0 & 0 & $\frac{-1}{15}$ & $\frac{16}{15}$ & 0 & 0 & $\frac{7}{8}$ & 2 & 8 & 1 & 3 & $\frac{5}{2}$ & 1 & $\frac{-1}{2}$ \\
\hline 2141 & $\frac{1}{960}$ & $\frac{-1}{960}$ & 0 & 0 & $\frac{-1}{15}$ & $\frac{16}{15}$ & 0 & 0 & $\frac{3}{4}$ & 2 & 4 & 1 & 2 & 3 & 2 & -1 \\
\hline 2204 & $\frac{1}{1920}$ & $\frac{-1}{1920}$ & $\frac{1}{60}$ & $\frac{-17}{60}$ & $\frac{1}{5}$ & $\frac{16}{15}$ & 0 & 0 & $\frac{3}{8}$ & 3 & 12 & $\frac{3}{2}$ & 4 & 2 & 0 & 0 \\
\hline 2222 & $\frac{1}{960}$ & $\frac{-1}{960}$ & 0 & 0 & $\frac{-1}{15}$ & $\frac{16}{15}$ & 0 & 0 & $\frac{3}{4}$ & 2 & 12 & 1 & 4 & 2 & 0 & 0 \\
\hline
\end{tabular}


Table 3. (Theorem 2.3 (i)) (contd.)

\begin{tabular}{|c|c|c|c|c|c|c|c|c|c|c|c|c|c|c|c|c|}
\hline$i j k l$ & $c_{1}$ & $c_{2}$ & $c_{3}$ & $c_{4}$ & $c_{5}$ & $c_{6}$ & $c_{7}$ & $c_{8}$ & $c_{9}$ & $c_{10}$ & $c_{11}$ & $c_{12}$ & $c_{13}$ & $c_{14}$ & $c_{15}$ & $c_{16}$ \\
\hline 2303 & $\frac{1}{960}$ & $\frac{-1}{960}$ & $\frac{1}{60}$ & $\frac{-17}{60}$ & $\frac{1}{5}$ & $\frac{16}{15}$ & 0 & $\frac{-1}{2}$ & $\frac{1}{4}$ & 3 & 20 & $\frac{3}{2}$ & $\frac{11}{2}$ & 1 & 0 & 1 \\
\hline 2321 & $\frac{1}{480}$ & $\frac{-1}{480}$ & 0 & 0 & $\frac{-1}{15}$ & $\frac{16}{15}$ & 0 & 0 & $\frac{1}{2}$ & 2 & 12 & 1 & 4 & 2 & 0 & 0 \\
\hline 2402 & $\frac{1}{480}$ & $\frac{-1}{480}$ & 0 & 0 & $\frac{-1}{15}$ & $\frac{16}{15}$ & 0 & -1 & $\frac{1}{2}$ & 2 & 28 & 1 & 7 & 0 & 0 & 2 \\
\hline 2501 & $\frac{1}{240}$ & $\frac{-1}{240}$ & $\frac{-1}{30}$ & $\frac{17}{30}$ & $\frac{-3}{5}$ & $\frac{16}{15}$ & 0 & -1 & 1 & 0 & 28 & 0 & 7 & 0 & 0 & 2 \\
\hline 3014 & $\frac{1}{1920}$ & $\frac{1}{640}$ & $\frac{-17}{480}$ & $\frac{1}{30}$ & $\frac{-1}{15}$ & $\frac{16}{15}$ & $\frac{1}{16}$ & 0 & $\frac{9}{8}$ & $\frac{9}{2}$ & 4 & $\frac{27}{16}$ & 6 & 4 & 2 & -1 \\
\hline 3032 & $\frac{1}{960}$ & $\frac{1}{320}$ & $\frac{-17}{240}$ & $\frac{1}{15}$ & $\frac{-1}{15}$ & $\frac{16}{15}$ & $\frac{1}{8}$ & 0 & $\frac{5}{4}$ & 3 & 4 & $\frac{11}{8}$ & 6 & 4 & 2 & -1 \\
\hline 3113 & $\frac{1}{960}$ & $\frac{-1}{960}$ & 0 & 0 & $\frac{-1}{15}$ & $\frac{16}{15}$ & 0 & 0 & 1 & 5 & 16 & $\frac{7}{4}$ & 7 & 3 & 1 & 0 \\
\hline 3131 & $\frac{1}{480}$ & $\frac{-1}{480}$ & 0 & 0 & $\frac{-1}{15}$ & $\frac{16}{15}$ & 0 & 0 & 1 & 4 & 4 & $\frac{3}{2}$ & 6 & 4 & 2 & -1 \\
\hline 3212 & $\frac{1}{480}$ & $\frac{-1}{480}$ & 0 & 0 & $\frac{-1}{15}$ & $\frac{16}{15}$ & 0 & 0 & 1 & 4 & 28 & $\frac{3}{2}$ & 8 & 2 & 0 & 1 \\
\hline 3311 & $\frac{1}{240}$ & $\frac{-1}{240}$ & 0 & 0 & $\frac{-1}{15}$ & $\frac{16}{15}$ & 0 & 0 & 1 & 2 & 28 & 1 & 8 & 2 & 0 & 1 \\
\hline 4004 & $\frac{1}{960}$ & $\frac{1}{320}$ & $\frac{-13}{240}$ & $\frac{-13}{60}$ & $\frac{1}{5}$ & $\frac{16}{15}$ & 0 & 0 & $\frac{3}{4}$ & 9 & 12 & 3 & 12 & 4 & 0 & 0 \\
\hline 4022 & $\frac{1}{480}$ & $\frac{1}{160}$ & $\frac{-17}{120}$ & $\frac{2}{15}$ & $\frac{-1}{15}$ & $\frac{16}{15}$ & 0 & 0 & $\frac{3}{2}$ & 6 & 12 & 2 & 12 & 4 & 0 & 0 \\
\hline 4103 & $\frac{1}{480}$ & $\frac{-1}{480}$ & $\frac{1}{60}$ & $\frac{-17}{60}$ & $\frac{1}{5}$ & $\frac{16}{15}$ & 0 & $\frac{-1}{2}$ & $\frac{1}{2}$ & 9 & 36 & 3 & $\frac{27}{2}$ & 2 & 0 & 2 \\
\hline 4121 & $\frac{1}{240}$ & $\frac{-1}{240}$ & 0 & 0 & $\frac{-1}{15}$ & $\frac{16}{15}$ & 0 & 0 & 1 & 6 & 12 & 2 & 12 & 4 & 0 & 0 \\
\hline 4202 & $\frac{1}{240}$ & $\frac{-1}{240}$ & 0 & 0 & $\frac{-1}{15}$ & $\frac{16}{15}$ & 0 & -1 & 1 & 6 & 60 & 2 & 15 & 0 & 0 & 4 \\
\hline 4301 & $\frac{1}{120}$ & $\frac{-1}{120}$ & $\frac{-1}{30}$ & $\frac{17}{30}$ & $\frac{-3}{5}$ & $\frac{16}{15}$ & 0 & -1 & 2 & 0 & 60 & 0 & 15 & 0 & 0 & 4 \\
\hline 5012 & $\frac{1}{240}$ & $\frac{1}{240}$ & $\frac{-17}{120}$ & $\frac{2}{15}$ & $\frac{-1}{15}$ & $\frac{16}{15}$ & $\frac{-1}{4}$ & 0 & $\frac{3}{2}$ & 10 & 44 & $\frac{11}{4}$ & 20 & 2 & -4 & 3 \\
\hline 5111 & $\frac{1}{120}$ & $\frac{-1}{120}$ & 0 & 0 & $\frac{-1}{15}$ & $\frac{16}{15}$ & 0 & 0 & 1 & 6 & 44 & 2 & 20 & 2 & -4 & 3 \\
\hline 6002 & $\frac{1}{120}$ & $\frac{-1}{120}$ & 0 & 0 & $\frac{-1}{15}$ & $\frac{16}{15}$ & $\frac{-1}{2}$ & -1 & 1 & 14 & 124 & $\frac{7}{2}$ & 31 & -4 & -8 & 10 \\
\hline 6101 & $\frac{1}{60}$ & $\frac{-1}{60}$ & $\frac{-1}{30}$ & $\frac{17}{30}$ & $\frac{-3}{5}$ & $\frac{16}{15}$ & 0 & -1 & 2 & 0 & 124 & 0 & 31 & -4 & -8 & 10 \\
\hline
\end{tabular}


Table 4. (Theorem 2.3 (ii))

\begin{tabular}{|c|c|c|c|c|c|c|c|c|c|c|c|c|c|c|c|c|}
\hline$i j k l$ & $d_{1}$ & $d_{2}$ & $d_{3}$ & $d_{4}$ & $d_{5}$ & $d_{6}$ & $d_{7}$ & $d_{8}$ & $d_{9}$ & $d_{10}$ & $d_{11}$ & $d_{12}$ & $d_{13}$ & $d_{14}$ & $d_{15}$ & $d_{16}$ \\
\hline 1007 & $\frac{1}{88}$ & $\frac{-1}{88}$ & $\frac{2}{11}$ & $\frac{1}{88}$ & $\frac{-1}{11}$ & $\frac{16}{11}$ & $\frac{1}{88}$ & $\frac{1}{88}$ & $\frac{43}{176}$ & $\frac{43}{22}$ & $\frac{8}{11}$ & $\frac{129}{176}$ & $\frac{-43}{44}$ & $\frac{-4}{11}$ & $\frac{43}{44}$ & $\frac{43}{44}$ \\
\hline 1025 & 0 & 0 & $\frac{2}{11}$ & $\frac{1}{44}$ & $\frac{-2}{11}$ & $\frac{32}{11}$ & 0 & $\frac{1}{44}$ & $\frac{7}{22}$ & $\frac{43}{22}$ & $\frac{12}{11}$ & $\frac{29}{44}$ & $\frac{-14}{11}$ & $\frac{20}{11}$ & $\frac{43}{44}$ & $\frac{14}{11}$ \\
\hline 1043 & 0 & 0 & $\frac{2}{11}$ & $\frac{1}{22}$ & $\frac{-4}{11}$ & $\frac{64}{11}$ & 0 & $\frac{1}{22}$ & $\frac{17}{44}$ & $\frac{21}{11}$ & $\frac{20}{11}$ & $\frac{25}{44}$ & $\frac{-17}{11}$ & $\frac{24}{11}$ & $\frac{21}{22}$ & $\frac{17}{11}$ \\
\hline 1061 & 0 & 0 & $\frac{2}{11}$ & $\frac{1}{11}$ & $\frac{-8}{11}$ & $\frac{128}{11}$ & 0 & $\frac{1}{11}$ & $\frac{3}{11}$ & $\frac{20}{11}$ & $\frac{-8}{11}$ & $\frac{7}{11}$ & $\frac{-12}{11}$ & $\frac{32}{11}$ & $\frac{10}{11}$ & $\frac{12}{11}$ \\
\hline 1106 & 0 & 0 & $\frac{2}{11}$ & $\frac{1}{44}$ & 0 & 0 & $\frac{1}{44}$ & 0 & $\frac{3}{44}$ & $\frac{5}{2}$ & $\frac{48}{11}$ & $\frac{10}{11}$ & 1 & $\frac{-28}{11}$ & $\frac{43}{44}$ & $\frac{37}{22}$ \\
\hline 1124 & 0 & 0 & $\frac{2}{11}$ & $\frac{1}{22}$ & 0 & 0 & 0 & 0 & $\frac{3}{22}$ & 2 & $\frac{48}{11}$ & $\frac{9}{11}$ & 1 & $\frac{16}{11}$ & 1 & 2 \\
\hline 1142 & 0 & 0 & $\frac{2}{11}$ & $\frac{1}{11}$ & 0 & 0 & 0 & 0 & $\frac{3}{11}$ & 2 & $\frac{48}{11}$ & $\frac{7}{11}$ & 0 & $\frac{16}{11}$ & 1 & 2 \\
\hline 1205 & $\frac{-1}{44}$ & $\frac{1}{44}$ & $\frac{2}{11}$ & $\frac{1}{22}$ & 0 & 0 & $\frac{1}{44}$ & 0 & $\frac{-3}{88}$ & $\frac{67}{22}$ & $\frac{92}{11}$ & $\frac{89}{88}$ & $\frac{59}{22}$ & $\frac{-28}{11}$ & $\frac{43}{44}$ & $\frac{59}{22}$ \\
\hline 1223 & 0 & 0 & $\frac{2}{11}$ & $\frac{1}{11}$ & 0 & 0 & 0 & 0 & $\frac{1}{44}$ & 2 & $\frac{92}{11}$ & $\frac{39}{44}$ & 3 & $\frac{16}{11}$ & 1 & 3 \\
\hline 1241 & 0 & 0 & $\frac{2}{11}$ & $\frac{2}{11}$ & 0 & 0 & 0 & 0 & $\frac{1}{22}$ & 2 & $\frac{48}{11}$ & $\frac{17}{22}$ & 2 & $\frac{16}{11}$ & 1 & 2 \\
\hline 1304 & $\frac{-1}{22}$ & $\frac{1}{22}$ & $\frac{2}{11}$ & $\frac{1}{11}$ & 0 & 0 & 0 & 0 & $\frac{-3}{44}$ & $\frac{34}{11}$ & $\frac{136}{11}$ & $\frac{45}{44}$ & $\frac{48}{11}$ & $\frac{16}{11}$ & 1 & 4 \\
\hline 1322 & 0 & 0 & $\frac{2}{11}$ & $\frac{2}{11}$ & 0 & 0 & 0 & 0 & $\frac{1}{22}$ & 2 & $\frac{136}{11}$ & $\frac{17}{22}$ & 4 & $\frac{16}{11}$ & 1 & 4 \\
\hline 1403 & $\frac{-1}{22}$ & $\frac{1}{22}$ & $\frac{2}{11}$ & $\frac{2}{11}$ & 0 & 0 & $\frac{-1}{22}$ & 0 & $\frac{-1}{22}$ & $\frac{23}{11}$ & $\frac{180}{11}$ & $\frac{10}{11}$ & $\frac{70}{11}$ & $\frac{104}{11}$ & $\frac{23}{22}$ & $\frac{62}{11}$ \\
\hline 1421 & 0 & 0 & $\frac{2}{11}$ & $\frac{4}{11}$ & 0 & 0 & 0 & 0 & $\frac{1}{11}$ & 2 & $\frac{136}{11}$ & $\frac{6}{11}$ & 4 & $\frac{16}{11}$ & 1 & 4 \\
\hline 1502 & 0 & 0 & $\frac{2}{11}$ & $\frac{4}{11}$ & 0 & 0 & $\frac{-1}{11}$ & 0 & $\frac{1}{11}$ & 0 & $\frac{224}{11}$ & $\frac{6}{11}$ & 8 & $\frac{192}{11}$ & $\frac{12}{11}$ & $\frac{80}{11}$ \\
\hline 1601 & $\frac{1}{11}$ & $\frac{-1}{11}$ & $\frac{2}{11}$ & $\frac{8}{11}$ & 0 & 0 & $\frac{-1}{11}$ & 0 & $\frac{4}{11}$ & $\frac{-24}{11}$ & $\frac{224}{11}$ & $\frac{-2}{11}$ & $\frac{80}{11}$ & $\frac{192}{11}$ & $\frac{12}{11}$ & $\frac{80}{11}$ \\
\hline 2015 & 0 & 0 & $\frac{2}{11}$ & $\frac{1}{22}$ & 0 & 0 & 0 & $\frac{1}{22}$ & $\frac{7}{11}$ & 5 & $\frac{92}{11}$ & $\frac{29}{22}$ & 0 & $\frac{-28}{11}$ & $\frac{43}{22}$ & $\frac{28}{11}$ \\
\hline 2033 & 0 & 0 & $\frac{2}{11}$ & $\frac{1}{11}$ & 0 & 0 & 0 & $\frac{1}{11}$ & $\frac{17}{22}$ & 4 & $\frac{92}{11}$ & $\frac{25}{22}$ & 0 & $\frac{16}{11}$ & $\frac{21}{11}$ & $\frac{34}{11}$ \\
\hline 2051 & 0 & 0 & $\frac{2}{11}$ & $\frac{2}{11}$ & 0 & 0 & 0 & $\frac{\overline{1}}{11}$ & $\frac{6}{11}$ & 4 & $\frac{48}{11}$ & $\frac{14}{11}$ & 0 & $\frac{16}{11}$ & $\frac{20}{11}$ & $\frac{24}{11}$ \\
\hline
\end{tabular}


Table 4. (Theorem 2.3 (ii))(contd.)

\begin{tabular}{|c|c|c|c|c|c|c|c|c|c|c|c|c|c|c|c|c|}
\hline$i j k l$ & $d_{1}$ & $d_{2}$ & $d_{3}$ & $d_{4}$ & $d_{5}$ & $d_{6}$ & $d_{7}$ & $d_{8}$ & $d_{9}$ & $d_{10}$ & $d_{11}$ & $d_{12}$ & $d_{13}$ & $d_{14}$ & $d_{15}$ & $d_{16}$ \\
\hline 2114 & 0 & 0 & $\frac{2}{11}$ & $\frac{1}{11}$ & 0 & 0 & 0 & 0 & $\frac{3}{11}$ & 5 & $\frac{136}{11}$ & $\frac{18}{11}$ & 3 & $\frac{16}{11}$ & 2 & 4 \\
\hline 2132 & 0 & 0 & $\frac{2}{11}$ & $\frac{2}{11}$ & 0 & 0 & 0 & 0 & $\frac{6}{11}$ & 4 & $\frac{136}{11}$ & $\frac{14}{11}$ & 2 & $\frac{16}{11}$ & 2 & 4 \\
\hline 2213 & 0 & 0 & $\frac{2}{11}$ & $\frac{2}{11}$ & 0 & 0 & 0 & 0 & $\frac{1}{22}$ & 4 & $\frac{180}{11}$ & $\frac{39}{22}$ & 6 & $\frac{104}{11}$ & 2 & 6 \\
\hline 2231 & 0 & 0 & $\frac{2}{11}$ & $\frac{4}{11}$ & 0 & 0 & 0 & 0 & $\frac{1}{11}$ & 4 & $\frac{136}{11}$ & $\frac{17}{11}$ & 4 & $\frac{16}{11}$ & 2 & 4 \\
\hline 2312 & 0 & 0 & $\frac{2}{11}$ & $\frac{4}{11}$ & 0 & 0 & 0 & 0 & $\frac{1}{11}$ & 2 & $\frac{224}{11}$ & $\frac{17}{11}$ & 8 & $\frac{192}{11}$ & 2 & 8 \\
\hline 2411 & 0 & 0 & $\frac{2}{11}$ & $\frac{8}{11}$ & 0 & 0 & 0 & 0 & $\frac{2}{11}$ & 0 & $\frac{224}{11}$ & $\frac{12}{11}$ & 8 & $\frac{192}{11}$ & 2 & 8 \\
\hline 3005 & $\frac{-1}{44}$ & $\frac{1}{44}$ & $\frac{2}{11}$ & $\frac{1}{11}$ & $\frac{4}{11}$ & $\frac{-64}{11}$ & $\frac{1}{44}$ & $\frac{1}{22}$ & $\frac{53}{88}$ & $\frac{201}{22}$ & $\frac{252}{11}$ & $\frac{205}{88}$ & $\frac{115}{22}$ & $\frac{-124}{11}$ & $\frac{129}{44}$ & $\frac{115}{22}$ \\
\hline 3023 & 0 & 0 & $\frac{2}{11}$ & $\frac{2}{11}$ & $\frac{8}{11}$ & $\frac{-128}{11}$ & 0 & $\frac{1}{11}$ & $\frac{35}{44}$ & $\frac{68}{11}$ & $\frac{236}{11}$ & $\frac{89}{44}$ & $\frac{67}{11}$ & 0 & $\frac{32}{11}$ & $\frac{67}{11}$ \\
\hline 3041 & 0 & 0 & $\frac{2}{11}$ & $\frac{4}{11}$ & $\frac{16}{11}$ & $\frac{-256}{11}$ & 0 & $\frac{2}{11}$ & $\frac{13}{22}$ & $\frac{70}{11}$ & $\frac{160}{11}$ & $\frac{45}{22}$ & $\frac{46}{11}$ & $\frac{-16}{11}$ & $\frac{31}{11}$ & $\frac{46}{11}$ \\
\hline 3104 & $\frac{-1}{22}$ & $\frac{1}{22}$ & $\frac{2}{11}$ & $\frac{2}{11}$ & 0 & 0 & 0 & 0 & $\frac{9}{44}$ & $\frac{100}{11}$ & $\frac{312}{11}$ & $\frac{117}{44}$ & $\frac{92}{11}$ & $\frac{16}{11}$ & 3 & 8 \\
\hline 3122 & 0 & 0 & $\frac{2}{11}$ & $\frac{4}{11}$ & 0 & 0 & 0 & 0 & $\frac{13}{22}$ & 6 & $\frac{312}{11}$ & $\frac{45}{22}$ & 8 & $\frac{16}{11}$ & 3 & 8 \\
\hline 3203 & $\frac{-1}{22}$ & $\frac{1}{22}$ & $\frac{2}{11}$ & $\frac{4}{11}$ & 0 & 0 & $\frac{-1}{22}$ & 0 & 0 & $\frac{67}{11}$ & $\frac{356}{11}$ & $\frac{59}{22}$ & $\frac{136}{11}$ & $\frac{280}{11}$ & $\frac{67}{22}$ & $\frac{128}{11}$ \\
\hline 3221 & 0 & 0 & $\frac{2}{11}$ & $\frac{8}{11}$ & 0 & 0 & 0 & 0 & $\frac{2}{11}$ & 6 & $\frac{312}{11}$ & $\frac{23}{11}$ & 8 & $\frac{16}{11}$ & 3 & 8 \\
\hline 3302 & 0 & 0 & $\frac{2}{11}$ & $\frac{8}{11}$ & 0 & 0 & $\frac{-1}{11}$ & 0 & $\frac{2}{11}$ & 0 & $\frac{400}{11}$ & $\frac{23}{11}$ & 16 & $\frac{544}{11}$ & $\frac{34}{11}$ & $\frac{168}{11}$ \\
\hline 3401 & $\frac{1}{11}$ & $\frac{-1}{11}$ & $\frac{2}{11}$ & $\frac{16}{11}$ & 0 & 0 & $\frac{-1}{11}$ & 0 & $\frac{6}{11}$ & $\frac{-68}{11}$ & $\frac{400}{11}$ & $\frac{10}{11}$ & $\frac{168}{11}$ & $\frac{544}{11}$ & $\frac{34}{11}$ & $\frac{168}{11}$ \\
\hline 4013 & 0 & 0 & $\frac{2}{11}$ & $\frac{4}{11}$ & $\frac{16}{11}$ & $\frac{-256}{11}$ & 0 & 0 & $\frac{1}{11}$ & $\frac{92}{11}$ & $\frac{468}{11}$ & $\frac{39}{11}$ & $\frac{200}{11}$ & $\frac{72}{11}$ & 4 & 12 \\
\hline 4031 & 0 & 0 & $\frac{2}{11}$ & $\frac{8}{11}$ & $\frac{32}{11}$ & $\frac{-512}{11}$ & 0 & 0 & $\frac{2}{11}$ & $\frac{96}{11}$ & $\frac{360}{11}$ & $\frac{34}{11}$ & $\frac{136}{11}$ & $\frac{-48}{11}$ & 4 & 8 \\
\hline 4112 & 0 & 0 & $\frac{2}{11}$ & $\frac{8}{11}$ & 0 & 0 & 0 & 0 & $\frac{2}{11}$ & 6 & $\frac{576}{11}$ & $\frac{34}{11}$ & 20 & $\frac{192}{11}$ & 4 & 16 \\
\hline 4211 & 0 & 0 & $\frac{2}{11}$ & $\frac{16}{11}$ & 0 & 0 & 0 & 0 & $\frac{4}{11}$ & 4 & $\frac{576}{11}$ & $\frac{24}{11}$ & 16 & $\frac{192}{11}$ & 4 & 16 \\
\hline 5003 & $\frac{-1}{22}$ & $\frac{1}{22}$ & $\frac{2}{11}$ & $\frac{8}{11}$ & $\frac{16}{11}$ & $\frac{-256}{11}$ & $\frac{-1}{22}$ & $\frac{-2}{11}$ & $\frac{-31}{22}$ & $\frac{115}{11}$ & $\frac{820}{11}$ & $\frac{63}{11}$ & $\frac{402}{11}$ & $\frac{424}{11}$ & $\frac{115}{22}$ & $\frac{258}{11}$ \\
\hline 5021 & 0 & 0 & $\frac{2}{11}$ & $\frac{16}{11}$ & $\frac{32}{11}$ & $\frac{-512}{11}$ & 0 & $\frac{-4}{11}$ & $\frac{-7}{11}$ & $\frac{118}{11}$ & $\frac{712}{11}$ & $\frac{46}{11}$ & $\frac{268}{11}$ & $\frac{-48}{11}$ & $\frac{9}{11}$ & $\frac{172}{11}$ \\
\hline 5102 & 0 & 0 & $\frac{2}{11}$ & $\frac{16}{11}$ & 0 & 0 & $\frac{-1}{11}$ & 0 & $\frac{-7}{11}$ & 0 & $\frac{928}{11}$ & $\frac{46}{11}$ & 40 & $\frac{896}{11}$ & $\frac{56}{11}$ & $\frac{344}{11}$ \\
\hline
\end{tabular}




\section{ACKNOWLEDGEMENTS}

We have used the open-source mathematics software SAGE (www.sagemath.org) to perform our calculations. Part of the work was done when the last named author visited the School of Mathematical Sciences, NISER, Bhubaneswar. He thanks the school for their hospitality and support.

\section{REFERENCES}

[1] A. Alaca, S. Alaca and K. S. Williams, Evaluation of the convolution sums $\sum_{l+18 m=n} \sigma(l) \sigma(m)$ and $\sum_{2 l+9 m=n} \sigma(l) \sigma(m)$, Int. Math. Forum 2 (2007), no. 1-4, 45-68.

[2] A. Alaca, S. Alaca and K. S. Williams, Evaluation of the convolution sums $\sum_{l+24 m=n} \sigma(l) \sigma(m)$ and $\sum_{3 l+8 m=n} \sigma(l) \sigma(m)$, Math. J. Okayama Univ. 49 (2007), 93-111.

[3] A. Alaca, S. Alaca and K. S. Williams, Seven octonary quadratic forms, Acta Arithmetica 135 (2008), 339-350.

[4] A. Alaca, S. Alaca and K. S. Williams, Fourteen octonary quadratic forms, Int. J. Number Theory 6 (2010), 37-50.

[5] S. Alaca and K. S. Williams, Evaluation of the convolution sums $\sum_{l+6 m=n} \sigma(l) \sigma(m)$ and $\sum_{2 l+3 m=n} \sigma(l) \sigma(m)$, J. Number Theory 124 (2007), no. 2, 491-510.

[6] G. E. Andrews and B. C. Berndt, Ramanujan's Lost Notebook, Part I, Springer, New York, 2005.

[7] B. C. Berndt, Ramanujan's Notebooks, Part III, Springer, New York, 1991.

[8] H. H. Chan and S. Cooper, Powers of theta functions, Pacific J. Math. 235 (2008) 1-14.

[9] H. H. Chan and Y. L. Ong, On Eisenstein series and $\sum_{m, n=-\infty}^{\infty} q^{m^{2}+m n+2 n^{2}}$, Proc. Amer. Math. Soc. 127 (1999) $1735-1744$.

[10] S. Cooper and D. Ye, Level 14 and 15 analogues of Ramanujan's elliptic functions to alternative bases, to appear in Trans. Amer. Math. Soc.; http://dx.doi.org/10.1090/tran6658

[11] J. G. Huard, Z. M. Ou, B. K. Spearman and K. S. Williams, Elementary evaluation of certain convolution sums involving divisor functions, in Number Theory for the Millennium, II (A. K. Peters, Natick, MA, 2002), pp. 229-274.

[12] N. Koblitz, Introduction to elliptic curves and modular forms, Second Edition, Graduate Texts in Mathematics 97, Springer, 1993.

[13] G. A. Lomadze, Representation of numbers by sums of the quadratic forms $x_{1}^{2}+x_{1} x_{2}+x_{2}^{2}$, Acta Arith. 54 (1989) 9-36.

[14] T. Miyake, Modular forms, Springer-Verlag, Berlin, 1989.

[15] E. Ntienjem, Evaluation of the convolution sum involving the sum of divisors function for 22, 44 and 52, Open Math. 15 (2017), 446-458.

[16] B. Ramakrishnan and Brundaban Sahu, Evaluation of convolution sums and some remarks on cusp forms of weight 4 and level 12, Math. J. Okayama Univ. 59 (2017), 71-79.

[17] B. Ramakrishnan, Brundaban Sahu and Anup Kumar Singh, On the Number of Representations by Certain Octonary Quadratic Forms With Coefficients 1, 2, 3, 4 and 6, Int. J. Number Theory (to appear). arXiv:1607.03809v3

[18] E. Royer, Evaluating convolution sums of the divisor function by quasimodular forms, Int. J. Number Theory $\mathbf{3}$ (2007), no. 2, 231-261.

[19] B. Schoeneberg, Elliptic Modular Functions: An Introduction, Die Grundlehren der Mathematischen Wissenschaften, Vol. 203, Springer, New York, 1974.

[20] G. Shimura, On modular forms of half-integral weight, Ann. Math. 97 (1973), 440-481.

[21] W. A. Stein, Modular Forms: A Computational Approach, American Mathematical Society, Providence, RI, 2007.

[22] K. S. Williams, On a double series of Chan and Ong, Georgian Math. J. 13 (2006) 793-805.

[23] Dongxi Ye, On the quaternary form $x^{2}+x y+7 y^{2}+z^{2}+z t+7 t^{2}$, Int. J. Number Theory 12 (2016), no. 7, 1791-1800.

(B. Ramakrishnan and Anup Kumar Singh) Harish-Chandra Research Institute, HBni, ChHatnag Road, Jhunsi, Allahabad - 211 019, India.

(Brundaban Sahu) School of Mathematical Sciences, National Institute of Science Education and Research, Bhubaneswar, HBNi, Via - Jatni, Khurda, Odisha - 752 050, India.

E-mail address, B. Ramakrishnan: ramki@hri.res.in

E-mail address, Brundaban Sahu: brundaban.sahu@niser.ac.in

E-mail address, Anup Kumar Singh: anupsingh@hri.res.in 